\title{
Optical properties and Zeeman spectroscopy of niobium in silicon carbide
}

\author{
Andreas Gällström, Björn Magnusson, Stefano Leone, Olle Kordina, Son Tien Nguyen,
} Viktor Ivády, Adam Gali, Igor Abrikosov, Erik Janzén and Ivan Gueorguiev Ivanov

\section{Linköping University Post Print}

\section{Tweet}

N.B.: When citing this work, cite the original article.

Original Publication:

Andreas Gällström, Björn Magnusson, Stefano Leone, Olle Kordina, Son Tien Nguyen, Viktor Ivády, Adam Gali, Igor Abrikosov, Erik Janzén and Ivan Gueorguiev Ivanov, Optical properties and Zeeman spectroscopy of niobium in silicon carbide, 2015, Physical Review B. Condensed Matter and Materials Physics, (92), 7, 075207.

http://dx.doi.org/10.1103/PhysRevB.92.075207

Copyright: American Physical Society

http://www.aps.org/

Postprint available at: Linköping University Electronic Press

http://urn.kb.se/resolve?urn=urn:nbn:se:liu:diva-122218 


\title{
Optical properties and Zeeman spectroscopy of niobium in silicon carbide
}

\author{
Andreas Gällström, ${ }^{1}$ Björn Magnusson, ${ }^{1}$ Stefano Leone, ${ }^{1}$ Olof Kordina, ${ }^{1}$ Nguyen T. Son, ${ }^{1}$ Viktor Ivády, ${ }^{1,2}$ Adam Gali, ${ }^{2,3}$ \\ Igor A. Abrikosov, ${ }^{1,4,5}$ Erik Janzén, ${ }^{1}$ and Ivan G. Ivanov ${ }^{1}$ \\ ${ }^{1}$ Linköping University, Department of Physics, Chemistry and Biology, S-581 83 Linköping, Sweden \\ ${ }^{2}$ Wigner Research Center for Physics, Hungarian Academy of Sciences, P.O. Box 49, H-1525 Budapest, Hungary \\ ${ }^{3}$ Department of Atomic Physics, Budapest University of Technology and Economics, Budafoki út. 8, H-1111 Budapest, Hungary \\ ${ }^{4}$ Materials Modeling and Development Laboratory, NUST “MISIS,” 119049 Moscow, Russia \\ ${ }^{5}$ LACOMAS Laboratory, Tomsk State University, 634050 Tomsk, Russia
}

(Received 29 January 2015; revised manuscript received 8 July 2015; published 27 August 2015)

\begin{abstract}
The optical signature of niobium in the low-temperature photoluminescence spectra of three common polytypes of $\mathrm{SiC}(4 \mathrm{H}, 6 \mathrm{H}$, and $15 \mathrm{R})$ is observed and confirms the previously suggested concept that $\mathrm{Nb}$ occupies preferably the $\mathrm{Si}-\mathrm{C}$ divacancy with both $\mathrm{Si}$ and $\mathrm{C}$ at hexagonal sites. Using this concept we propose a model considering a $\mathrm{Nb}$-bound exciton, the recombination of which is responsible for the observed luminescence. The exciton energy is estimated using first-principles calculation and the result is in very good agreement with the experimentally observed photon energy in $4 \mathrm{H} \mathrm{SiC}$ at low temperature. The appearance of six Nb-related lines in the spectra of the hexagonal $4 \mathrm{H}$ and $6 \mathrm{H}$ polytypes at higher temperatures is tentatively explained on the grounds of the proposed model and the concept that the $\mathrm{Nb}$ center can exist in both $\mathrm{C}_{1 h}$ and $\mathrm{C}_{3 v}$ symmetries. The Zeeman splitting of the photoluminescence lines is also recorded in two different experimental geometries and the results are compared with theory based on phenomenological Hamiltonians. Our results show that $\mathrm{Nb}$ occupying the divacancy at the hexagonal site in the studied $\mathrm{SiC}$ polytypes behaves like a deep acceptor.
\end{abstract}

DOI: 10.1103/PhysRevB.92.075207

PACS number(s): 78.55.-m, 71.55.-i, 71.70.Ej

\section{INTRODUCTION}

It is well known that silicon carbide exists in many different polytypes. Among these the simplest is the cubic SiC (3C-SiC, zinc-blende structure) and all other polytypes can be viewed as differing by the stacking sequence of the $\mathrm{Si}-\mathrm{C}$ atomic biplanes along the [111] direction of 3C-SiC. With the exception of $3 \mathrm{C}-\mathrm{SiC}$ and $2 \mathrm{H}-\mathrm{SiC}$ (würtzite structure) all other polytypes possess different numbers of inequivalent sites for both $\mathrm{Si}$ and $\mathrm{C}$ within their unit cell, hence one and the same substitutional atom replacing a host atom at different inequivalent lattice sites exhibits different properties. Three common polytypes, $4 \mathrm{H}-$ $\mathrm{SiC}, 6 \mathrm{H}-\mathrm{SiC}$, and $15 \mathrm{R}-\mathrm{SiC}$ are considered in the present study, all of them displaying different combinations of hexagonal and cubic (a.k.a. quasicubic) inequivalent lattice sites. [In common terminology a site is referred to as hexagonal or cubic if the stacking arrangement at this site mimics the arrangement of the hexagonal (würtzite) or the cubic (zinc-blende) structure, respectively.] Thus, $4 \mathrm{H}-\mathrm{SiC}$ and $6 \mathrm{H}-\mathrm{SiC}$ (crystals of hexagonal symmetry) both have one hexagonal site per unit cell (for Si or $\mathrm{C})$, but differ in the number of cubic sites, one in $4 \mathrm{H}-\mathrm{SiC}$ and two in 6H-SiC. 15R-SiC (rhombohedral crystal symmetry) has two hexagonal and three cubic inequivalent lattice sites.

Due to the site dependence of the properties of a substitutional impurity, i.e., an impurity atom replacing a host atom (e.g., $\mathrm{Si}$ ), the number of optical centers observed in photoluminescence (PL) is expected to be equal to the number of inequivalent sites. This is clearly demonstrated in the case of incorporation of transition metals from the first row of the periodic table in $\mathrm{SiC}$, which can be seen from optical studies on, for instance, vanadium [1-5], chromium [5,6], and titanium [5,7-9]. All these elements replace $\mathrm{Si}$ atoms due to their rather large atomic radii. However, optical and electron spin resonance studies of $\mathrm{SiC}$ doped with tungsten (W) [10], Mo [5,11-13], and $\mathrm{Nb}$ (considered in this work) show that at least for some transition metals from the second and the third row of the periodic table the number of optical centers does not follow the number of inequivalent sites, which suggests that these elements might incorporate in $\mathrm{SiC}$ in a configuration different from the mere substitutional one. In the case of $\mathrm{W}$, for example, the number of PL centers observed in different polytypes equals the number of quasicubic sites [10]. There exist also several other unidentified defects under discussion in the literature; for example, UD-2 [14] and UD-3 [15], for which the number of optical centers differs from the number of inequivalent lattice sites. Thus, the number of optical centers associated with UD-2 replicates the number of possible nearest-neighbor pair configurations, while for UD-3 this number follows the number of inequivalent hexagonal sites in different polytypes.

In a recent work [16], new theoretical results on transition metal incorporation in $\mathrm{SiC}$ were published. Several transition metals from the first, second, and third rows of the periodic table ( $\mathrm{Ti}, \mathrm{V}, \mathrm{Cr}, \mathrm{Nb}, \mathrm{Mo}$, and $\mathrm{W}$ ) are considered in this work, and first-principles calculations have been carried out for two possible configurations of the defect: pure substitutional on Si site (denoted as $M_{\mathrm{Si}}$, where $M$ is the corresponding transition metal), and the so-called asymmetric split-vacancy (ASV) configuration which can be denoted $M_{\mathrm{Si}}-V_{\mathrm{C}}$, where $V_{\mathrm{C}}$ denotes the carbon vacancy. The latter case can be viewed as the impurity atom $M$ occupying the silicon-carbon divacancy $V_{\mathrm{Si}}-V_{\mathrm{C}}$ with $M$ shifted closer to the $\mathrm{Si}$ site, hence the asymmetry. A comparison of the formation energies of the ASV configuration $\left(M_{\mathrm{Si}}-V_{\mathrm{C}}\right)$ and pure substitutional $\left(M_{\mathrm{Si}}\right)$ shows that for the metals from the first row (Ti, V, Cr) the $M_{\mathrm{Si}}$ configuration is strongly prevailing (it has significantly lower formation energy). On the other hand, for the rest of the metals considered $(\mathrm{Nb}, \mathrm{Mo}, \mathrm{W})$ one of the possible ASV configurations and the substitutional configuration have similar formation energies, but the ASV configuration is 
favored. In the case of $\mathrm{Nb}$, it was found that maximum overlap between the dangling bonds of the Si atoms and the $d$ orbitals of the $\mathrm{Nb}$ atom occurs in the ASV hexagonal-hexagonal (h-h) configuration (i.e., the vacant $\mathrm{C}$ and $\mathrm{Si}$ sites are hexagonal nearest neighbors aligned along the crystal $c$ axis). Thus, this configuration (denoted hereafter as ASV h-h) is expected to form in significantly larger concentrations than any other. Electron spin resonance (ESR) measurements are consistent with the ASV h-h configuration for the case of $\mathrm{Nb}$-doped 4H-SiC [16] (more details on the ESR spectra are presented in [17]). The observed ESR spectrum clearly shows the hyperfine interaction of an $S=1 / 2$ spin with two $\mathrm{Si}$ atoms, demonstrating also the Jahn-Teller distortion to $\mathrm{C}_{1 h}$ symmetry, in accord with the calculation.

In this work we give a detailed description of the optical transitions assigned to $\mathrm{Nb}$-related centers in $\mathrm{SiC}$, propose a model for their origin, and compare the experimental results with the results from first-principles theoretical calculation. (A preliminary account on some of the experimental data and the model was presented in [18]). We consider also the Zeeman splitting of the Nb-related lines on the grounds of phenomenological models and compare the experimental line splitting with the predictions of these models. Section II describes experimental details concerning the samples and the experimental setups used. Section III presents the optical signature of the $\mathrm{Nb}$ center in three polytypes of $\mathrm{SiC}$ and considers in more detail the temperature dependence and photoluminescence excitation (PLE) spectroscopy of the $\mathrm{Nb}$ lines revealing similar electronic structure of the defect in the hexagonal $4 \mathrm{H}$ and $6 \mathrm{H}$ polytypes. Section IV presents the model of a Nb-bound exciton and the results of first-principle calculations. Section V deals with the Zeeman effect on the $\mathrm{Nb}$ lines, presenting the experimental data and the models used for its interpretation. Finally, the conclusions are summarized in Section VI.

\section{SAMPLES AND EXPERIMENTAL DETAILS}

The SiC samples considered in this study are grown using high-temperature chemical vapor deposition (HTCVD) [19]. In order to confirm the association of the PL lines discussed further on with $\mathrm{Nb}$, doping experiment is carried out with the $4 \mathrm{H}-\mathrm{SiC}$ polytype by placing a $\mathrm{Nb}$ metallic flake upstream in the reactor during growth. The $\mathrm{Nb}$-doped sample was grown right after growing an undoped reference sample in a standard HTCVD reactor. Both samples are unintentionally doped $n$ type ( $\mathrm{N}$ doping in the $10^{14} \mathrm{~cm}^{-3}$ range). The outcome of the doping experiment is summarized in the inset of Fig. 1; the figure displays also the niobium-associated lines observed at low temperature in the three studied polytypes. The low-temperature ( $T=2 \mathrm{~K}$ ) PL spectrum of the Nb-doped sample shows a single line at $1.3825 \mathrm{eV}$, which is not observed in the reference sample grown in the previous run. Secondary ion mass spectrometry (SIMS) was performed on both samples confirming that $\mathrm{Nb}$ is indeed incorporated in concentration $\sim 9 \times 10^{15} \mathrm{~cm}^{-3}$ in the doped sample, whereas in the reference sample its concentration is below the detection limit of $\sim 2 \times 10^{13} \mathrm{~cm}^{-3}$ (see the inset in Fig. 1). On the grounds of this doping experiment we assign the observed PL line at 1.3825 $\mathrm{eV}(1382.5 \mathrm{meV})$ in $4 \mathrm{H}-\mathrm{SiC}$ to $\mathrm{Nb}$-related defect. We note

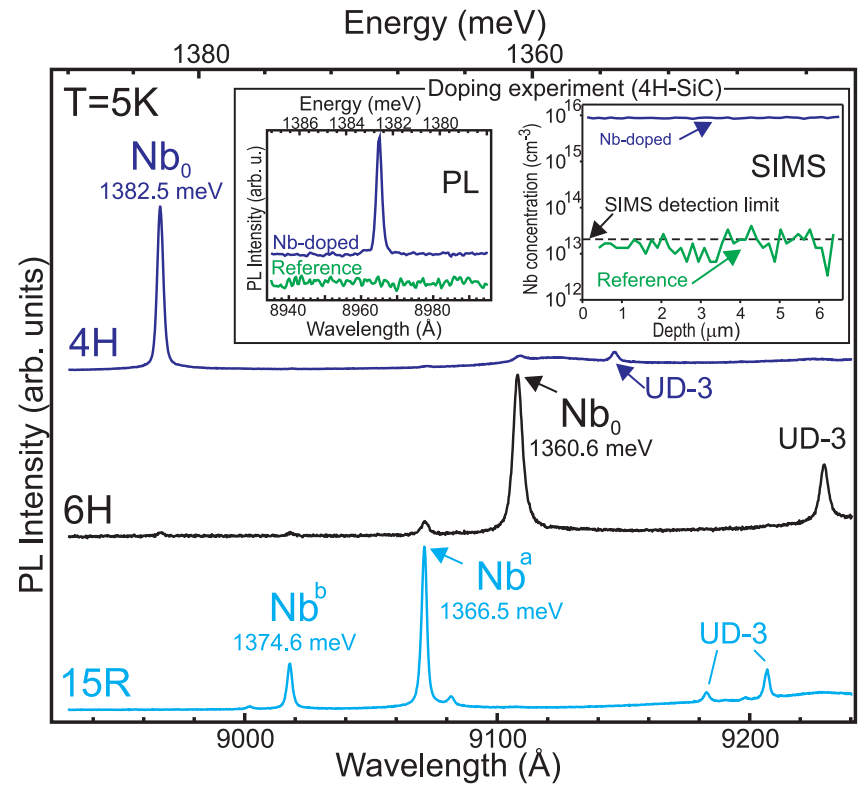

FIG. 1. (Color online) PL spectra of 4H-, 6H-, and 15R-SiC showing the $\mathrm{Nb}$-associated lines at $T=5 \mathrm{~K}$. The inset summarizes the results of the doping experiment discussed in text. The weak contribution of the $15 \mathrm{R}$ and $4 \mathrm{H} \mathrm{Nb}$-related lines in the spectrum of the $6 \mathrm{H}$ sample, as well as of the $\mathrm{Nb}$ line of $6 \mathrm{H}$ in the $4 \mathrm{H}$ spectrum are due to the presence of microscopic polytype inclusions in these samples. In particular, the $15 \mathrm{R}$ sample was obtained from a macroscopic inclusion in the $6 \mathrm{H}$ polytype. (Some data is adapted with permission from Ref. [18].)

that, to the best of our knowledge, this line is not among the commonly detected lines in as-grown samples [14]. However, this PL line is observed in semi-insulating (SI) $4 \mathrm{H}-\mathrm{SiC}$ samples grown in a reactor where the susceptor has parts made of niobium carbide ( $\mathrm{NbC})$. The concentration of $\mathrm{Nb}$ in these latter samples (including also $6 \mathrm{H}$ and $15 \mathrm{R}$ polytypes) as estimated by SIMS is somewhat higher, $\sim 3 \times 10^{16} \mathrm{~cm}^{-3}$, and the rest of the results presented here are from such unintentionally doped samples grown in a reactor with $\mathrm{NbC}$ susceptor parts. Figure 1 (except for the inset) also presents the spectra from these samples. The 15R-SiC sample was cut from a macroscopic (several millimeters large) inclusion in the $6 \mathrm{H}$ polytype, and the $15 \mathrm{R}$ polytype was verified by monitoring the near-band gap luminescence in several random points (not shown) at low temperature [20].

$\mathrm{Ar}^{+}$-ion laser ultraviolet lines (351 and/or $364 \mathrm{~nm}$ ) are used as above-band-gap excitation in the PL measurements. The luminescence is detected by either a monochromator coupled to a CCD camera or a double monochromator equipped with an InGaAsP photomultiplier tube, although the general lowtemperature spectra displayed in Fig. 1 are obtained using a Fourier-transform spectrometer. In all cases, the spectral resolution is by at least a factor of 2 better than the observed linewidth $(\sim 0.3 \mathrm{meV}$ at full width at half maximum for all $\mathrm{Nb}$ lines). PLE spectroscopy and the Zeeman measurements using fiber with strong damping in the ultraviolet are performed using a Ti:sapphire laser as an excitation source.

Zeeman measurements in magnetic fields perpendicular to the crystal $c$ axis $(\mathbf{B} \perp \mathbf{c})$ up to $10 \mathrm{~T}$ are performed using a cryostat coupled to an optical fiber, hence only the Faraday 
configuration is available (the direction of propagation of the detected light is parallel to the magnetic field). Due to weaker luminescence received through the fiber the spectral resolution has to be sacrificed in favor of the signal, which results in slight broadening of the $\mathrm{Nb}$ lines to $\sim 0.4 \mathrm{meV}$. The Zeeman effect in magnetic field parallel to the $c$ axis (B $\| \mathbf{c}$, up to $5 \mathrm{~T}$ ) is measured in another cryostat where also only Faraday configuration is available, but it is possible also to study the polarization of the lines. Left and right circularly polarized light is detected using an achromatic quarter-wave plate in combination with a linear polarizer. Finally, the decay time of the $\mathrm{Nb}$-related emission is measured using photon-counting and pulsed excitation at $355 \mathrm{~nm}$ (the third harmonic of a $\mathrm{Nd}$ :YAG laser) with a time resolution $\sim 1 \mathrm{~ns}$.

\section{OPTICAL PROPERTIES OF THE Nb CENTER}

A. Photoluminescence signature of $\mathrm{Nb}$ in $4 \mathrm{H}-, 6 \mathrm{H}-$, and $15 \mathrm{R}-\mathrm{SiC}$

The low-temperature PL spectra of $4 \mathrm{H}-, 6 \mathrm{H}-$, and $15 \mathrm{R}-\mathrm{SiC}$ are displayed in Fig. 1. (The PL line due to the commonly observed but unidentified defect UD-3 is also visible in the displayed region [15].) We notice that in the $4 \mathrm{H}$ and $6 \mathrm{H}$ polytypes we observe only one line associated with $\mathrm{Nb}$, whereas in $15 \mathrm{R}-\mathrm{SiC}$ there are two lines (denoted $\mathrm{Nb}^{\mathrm{a}}$ and $\mathrm{Nb}^{\mathrm{b}}$ in Fig. 1). Apparently, the number of optical centers corresponds to the number of inequivalent hexagonal sites in the unit cell of each polytype, in agreement with the theoretical expectation that $\mathrm{Nb}$ prefers the $\mathrm{ASV} h-\mathrm{h}$ configuration (at hexagonal site) in these three polytypes [16].

\section{B. Temperature dependence and PLE spectroscopy}

The temperature dependence of the Nb-related part of the $\mathrm{PL}$ spectra in $4 \mathrm{H}-\mathrm{SiC}$ recorded with polarization perpendicular to the $c$ axis $(\mathbf{E} \perp \mathbf{c})$ and parallel to it $(\mathbf{E} \| \mathbf{c})$ is displayed in Fig. 2(a). As the temperature increases, five new lines enumerated in order of increasing energy in the figure appear on the high-energy side of the low-temperature $\mathrm{Nb}_{0}$ line. The interplay of the relative intensities of the six lines with the temperature shows thermalization between sublevels of the excited (initial) state involved in the optical transition, whereas the ground (final) state behaves as a single energy level. The structure of the spectrum of $6 \mathrm{H}-\mathrm{SiC}$ is very similar, hence only the polarized spectra at $27 \mathrm{~K}$ are shown in Fig. 2(b). The PLE spectra detected at the $\mathrm{Nb}_{0}$ line for $4 \mathrm{H}$ and $6 \mathrm{H} \mathrm{SiC}$ are shown as the bottom curve in Figs. 2(a) and 2(b), respectively. We notice that the $\mathrm{Nb}_{5}$ line is hardly visible in the PL spectra of both polytypes due to its weakness, the proximity of the strong $\mathrm{Nb}_{4}$ line, and rapid temperature-induced broadening of the lines. Also, in the case of $6 \mathrm{H} \mathrm{SiC}$ the clear observation of $\mathrm{Nb}_{5}$ is impeded by the appearance of the $\mathrm{Nb}^{\mathrm{a}}$ line of $15 \mathrm{R}$, which is due to microscopic inclusions of this polytype in the $6 \mathrm{H}$ sample (cf. Fig. 1). We have plotted also one unpolarized spectrum for each polytype, chosen so as to show the $\mathrm{Nb}_{5}$ line as a weak shoulder on the high-energy side. [We note that $\mathrm{Nb}_{5}$ is better visible in the spectra detected along the $c$ axis (with $\mathbf{E} \perp \mathbf{c}$ polarization), e.g., in the Zeeman experiments with magnetic field parallel to the $c$ axis (cf. the spectra at zero field in Sec. IV), because the strong $\mathrm{Nb}_{4}$ line polarized $\mathbf{E} \| \mathbf{c}$ is completely suppressed.] However, $\mathrm{Nb}_{5}$ is clearly visible in

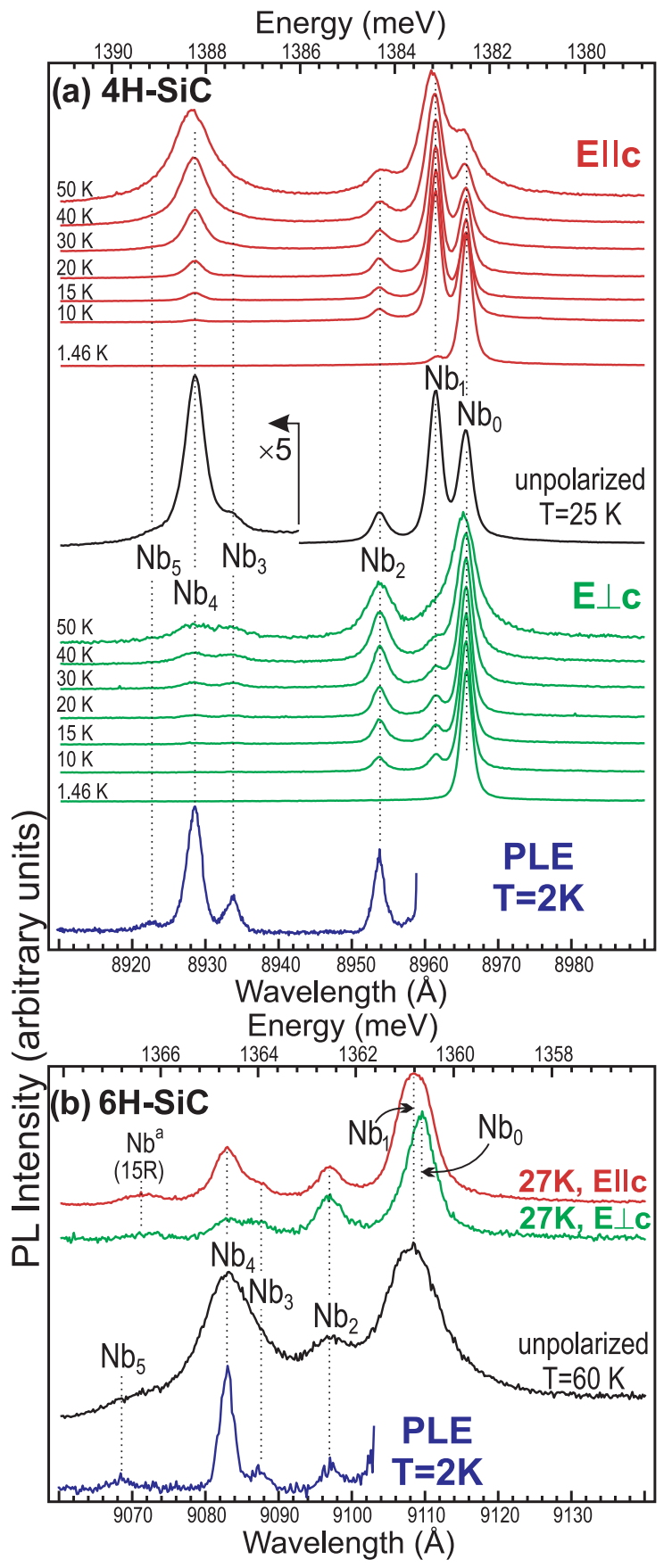

FIG. 2. (Color online) (a) Temperature dependence of the polarized PL spectra associated with $\mathrm{Nb}$ in $4 \mathrm{H}-\mathrm{SiC}$. (b) Polarized spectra of the $\mathrm{Nb}$-related spectrum in $6 \mathrm{H}-\mathrm{SiC}$ at $27 \mathrm{~K}$. The lowermost curve in both panels is the corresponding PLE spectrum. One unpolarized spectrum per polytype is also shown, chosen so as to illustrate the appearance as a shoulder of the very weak $\mathrm{Nb}_{5}$ line. (Some data is adapted with permission from Ref. [18].)

the PLE spectra for both polytypes [see the bottom curves in Figs. 2(a) and 2(b)], and its line positions displayed in Table I are obtained from these curves. Thus, the PLE spectra confirm that $\mathrm{Nb}_{0}-\mathrm{Nb}_{5}$ lines are related to the same center, although the $\mathrm{Nb}_{1}$ line, being too close to $\mathrm{Nb}_{0}$, falls in the Rayleigh wing of the laser and cannot be distinguished in PLE. Furthermore, $\mathrm{Nb}_{1}$ and $\mathrm{Nb}_{4}$ lines have predominantly 
TABLE I. Photon energies in meV (wavelength in $\AA$ in parentheses) of the six Nb-related transitions in $4 \mathrm{H}-$ and $6 \mathrm{H}-\mathrm{SiC}$. The polarization of each line is also shown ( $\|:$ parallel to the $c$ axis; $\perp$ : perpendicular to it).

\begin{tabular}{lccccc}
\hline \hline Polytype & $\begin{array}{c}\mathrm{Nb}_{0} \\
\text { and } \perp\end{array}$ & $\begin{array}{c}\mathrm{Nb}_{1} \\
\|\end{array}$ & $\begin{array}{c}\mathrm{Nb}_{2} \\
\text { and } \perp\end{array}$ & $\begin{array}{c}\mathrm{Nb}_{3} \\
\text { and } \perp\end{array}$ & $\begin{array}{c}\mathrm{Nb}_{4} \\
\|\end{array}$ \\
\hline 4H SiC & 1382.5 & 1383.15 & 1384.3 & 1387.4 & $\begin{array}{c}\mathrm{Nb}_{5} \\
\text { and } \perp\end{array}$ \\
& $(8965.5 \AA)$ & $(8961.4 \AA)$ & $(8953.9 \AA)$ & $(8933.9 \AA)$ & 1388.2 \\
$6 \mathrm{H} \mathrm{SiC}$ & 1360.6 & 1360.8 & 1362.5 & 1363.9 & $\begin{array}{c}(8928.8 \AA) \\
(89922.4 \AA) \\
\end{array}$ \\
\hline \hline
\end{tabular}

E $\|$ c polarization in both $4 \mathrm{H}-$ and $6 \mathrm{H}-\mathrm{SiC}$, while the rest of the lines do not exhibit preferable polarization. Note that in $6 \mathrm{H}-\mathrm{SiC}$ the $\mathrm{Nb}_{0}$ and $\mathrm{Nb}_{1}$ lines almost merge, but still can be distinguished owing to the peak shift observed with different polarizations [cf. Fig. 2(b)]. No additional lines appear at higher temperatures in either polytype, the only notable effect being broadening and quenching of the lines so that the whole emission vanishes around $190 \mathrm{~K}$.

The photon energies and wavelengths of the $\mathrm{Nb}$-related transitions in $4 \mathrm{H}$ and $6 \mathrm{H} \mathrm{SiC}$ are summarized in Table I together with their polarization. The structure of the spectrum in these two polytypes is very similar, indicating similar microscopic structure of the defect. In discussing our model below we will see that the structure of the Nb-related spectrum stems from the structure of the valence band, which is similar in the hexagonal $4 \mathrm{H}$ and $6 \mathrm{H} \mathrm{SiC}$ polytypes and it is sufficient to investigate in detail one of them. On the other hand, although there exist both experimental [21] and theoretical [22] studies on the valence-band structure of $15 \mathrm{R} \mathrm{SiC}$, the rhombohedral $15 \mathrm{R}$ polytype is substantially less explored than the hexagonal polytypes. Therefore, in the rest of this paper we shall consider in detail only the $4 \mathrm{H}-\mathrm{SiC}$ polytype.

\section{MODEL OF Nb-BOUND EXCITON}

According to the model outlined in Ref. [18], the PL lines due to $\mathrm{Nb}$ in the ASV h-h configuration are actually arising from recombination of an exciton bound to the defect. The main idea-a bound exciton consisting of a tightly bound electron and Coulombically bound hole-is corroborated here by a semiquantitative estimate of the exciton recombination energy obtained within a simple physical picture using previously calculated energies [16] of the Nb defect in different charge states. Results of new first-principles calculations treating the exciton bound at the niobium center in $4 \mathrm{H}-\mathrm{SiC}$ are also presented and shown to be in good agreement with both the semiquantitative estimates made in the next section and the experimental data. The selection rules for the optical transitions are also discussed.

\section{A. Mechanism of binding an exciton to the neutral $\mathrm{Nb}^{\circ}$ center}

According to the calculation of Ref. [16], the $\mathrm{Nb}$ center in ASV configuration can exist in neutral, positively charged and negatively charged states, all with energy levels within the energy band gap of $4 \mathrm{H}-\mathrm{SiC}$. First-principles calculations using the method described in [16] yield the following positions of these levels in the energy band gap of $4 \mathrm{H}-\mathrm{SiC}$ (all energies are relative to the valence-band edge). The positively charged state has an energy level $E_{\mathrm{Nb}}^{+}$about $0.2 \mathrm{eV}$, the neutral state has two energy levels at $E_{\mathrm{Nb}}^{\prime 0} \sim 0.4 \mathrm{eV}$ and $E_{\mathrm{Nb}}^{\prime \prime 0} \sim 0.6 \mathrm{eV}$ associated with the Jahn-Teller splitting of the one-electron $e$ level [16], and the negatively charged state has a level $E_{\mathrm{Nb}}^{-} \sim 0.9 \mathrm{eV}$, as depicted in Fig. 3(a).

We assume that without any photoexcitation the $\mathrm{Nb}$ center is in the neutral charge state, in agreement with the ESR results $[17,23]$. On the other hand, if an electron or hole is captured to form one of the charged states, this charged center

(a)
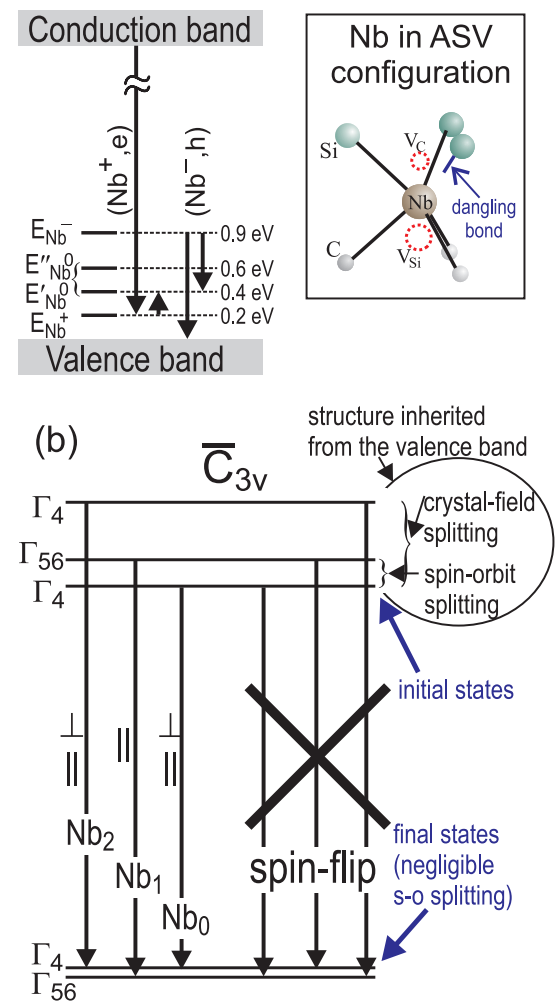

FIG. 3. (Color online) Schematic presentation of the energy levels discussed in text. (a) Energy levels of $\mathrm{Nb}$ in different charge states. The splitting of the neutral charge state is due to Jahn-Teller distortion of the defect. The down- (up-) arrows represent the processes discussed in text which contribute to (decrease) the recombination energy emitted as a photon. (b) Suggested structure of the ground and excited states of $\mathrm{Nb}$ in the ASV configuration. The selection rules for optical transitions are also depicted; the overstricken transitions are allowed by group theory but require spin flip, making them much less probable. The inset illustrates schematically the bonding in the neutral charge state of $\mathrm{Nb}$ in the ASV configuration. 
becomes a Coulomb center capable of capturing a particle of the opposite charge, a hole for $\mathrm{Nb}^{-}$or an electron for $\mathrm{Nb}^{+}$, respectively. Thus we obtain two possible neutral systems, which can be viewed as an exciton bound to the neutral $\mathrm{Nb}^{0}$ center. Using the calculated energy levels given above, it is possible to estimate roughly the binding energies of an exciton with a strongly bound hole and a weakly bound electron in the Coulomb field of $\mathrm{Nb}^{+}$[denoted for brevity $\left(\mathrm{Nb}^{+}, e\right)$ in Fig. 3(a)], and vice versa, an exciton with a strongly bound electron (forming $\mathrm{Nb}^{-}$) and weakly bound hole [denoted $\left(\mathrm{Nb}^{-}, h\right)$ in Fig. 3(a)]. Considering the weakly bound particle as free at the extremum of the corresponding (conduction or valence) energy band we use simple energy consideration in order to estimate the energy stored in the bound exciton which can be released as a photon. For example, in the case of a weakly bound electron to $\mathrm{Nb}^{+}$, the energy released during the recombination of the center into the $\mathrm{Nb}^{0}$ state is a sum of the energies $\left(E_{\mathrm{C}}-E_{\mathrm{Nb}}^{+}\right)$and $\left(E_{\mathrm{Nb}}^{+}-E_{\mathrm{Nb}}^{\prime 0}\right)$, where $E_{\mathrm{C}} \approx 3.2 \mathrm{eV}$ is the theoretically calculated energy of the band gap. [The corresponding energy quantities are illustrated by two vertical arrows for the case $\left(\mathrm{Nb}^{+}, e\right)$ in Fig. 3(a).] In this way we obtain that the binding energy of an exciton with strongly bound hole and weakly bound electron is of the order of $2.8 \mathrm{eV}$, whereas in the case of $\left(\mathrm{Nb}^{-}, h\right)$ the bound-exciton energy is $\sim 1.4 \mathrm{eV}$. The latter estimate is obtained by adding the energy $E_{\mathrm{Nb}}^{-}$released by a free hole recombining with $\mathrm{Nb}^{-}$and the energy $\left(E_{\mathrm{Nb}}^{-}-E_{\mathrm{Nb}}^{\prime 0}\right)$ which is further released in order to obtain $\mathrm{Nb}^{0}$ in the ground state, as illustrated in Fig. 3(a). These rough estimates demonstrate that the case of a hole weakly bound to $\mathrm{Nb}^{-}$is by far the energetically favorable one and, in addition, the theoretical estimate of the exciton recombination energy of $\sim 1.4 \mathrm{eV}$ is very close to the experimentally observed one $(\sim 1.38 \mathrm{eV})$. Thus, it is reasonable to consider the experimental results in the frame of a model of recombination of a bound exciton with a strongly bound electron and an effective-mass-like hole weakly bound in the Coulomb field of the negatively charged defect referred to hitherto as $\left(\mathrm{Nb}^{-}, h\right)$.

The mechanism of binding an exciton to $\mathrm{Nb}$ can be considered also from another point of view, as is done in Ref. [18] and outlined briefly here. The five valence electrons of the $\mathrm{Nb}$ atom are used to saturate five of the six dangling bonds of the divacancy (three from the silicon vacancy, and three from the carbon vacancy; cf. Fig. 1 of Ref. [16] and the inset in Fig. 3). Capturing one more electron (i.e., the electron from the exciton) into the remaining dangling bond of the carbon vacancy will complete the bonding of the $\mathrm{Nb}$ atom and form the negatively charged $\mathrm{Nb}^{-}$center. Saturated bonds can be regarded as an analog of a filled shell in an atom; hence the captured electron remains strongly localized at defect. The situation is similar to the well-known mechanism of binding excitons to isoelectronic defects, when one of the particles in the exciton experiences short-range attractive potential at the defect and the remaining particle is bound in the resulting Coulomb field of the charged defect. In the case of $\mathrm{Nb}$ in the ASV configuration the reason for strong binding of the electron is quite apparent, namely, saturation of the only remaining dangling bond. In order to cope with the selection rules, we treat the weakly bound hole as effective masslike (nearly free) and consider the structure of the valence band near its extremum.

\section{B. Selection rules for the optical transitions}

The symmetry of the possible exciton states will be treated considering separately the symmetries of the negatively charged defect and the weakly bound (effective-mass-like) hole. Due to the same crystallographic group $\left(\mathrm{C}_{6 v}^{4}\right)$ and similar band structure the analysis presented below is equally valid for $4 \mathrm{H}$ and $6 \mathrm{H} \mathrm{SiC}$. When an extra (12th) electron is captured to $\mathrm{Nb}$ thus completing the bonding the reason for Jahn-Teller splitting vanishes and the $\mathrm{Nb}^{-}$center relaxes to $\mathrm{C}_{3 v}$ symmetry (recall that the symmetry of $\mathrm{Nb}^{0}$ in its Jahn-Teller distorted configuration is $\mathrm{C}_{1 h}$ ) [16]. Group-theoretical consideration shows that the ground state of $\mathrm{Nb}^{-}$is a fully symmetric singlet transforming as the $\Gamma_{1}$ irreducible representation of $\mathrm{C}_{3 v}$, commonly denoted also as ${ }^{1} A_{1}$. The ground state of an effective-mass-like hole bound in the Coulomb field of $\mathrm{Nb}^{-}$ will exhibit three closely spaced components corresponding to threefold splitting of the uppermost valence band (due to crystal-field and spin-orbit splitting) [24]. For a free hole the proper symmetry would be $\mathrm{C}_{6 v}$, and the uppermost valence band transforms as the $\Gamma_{9}$ representation of the double group $\overline{\mathrm{C}}_{6 v}$ (the bar above the group symbol will denote hereafter the corresponding double group). The next valence band split off by spin-orbit interaction transforms as $\Gamma_{7}$, and finally, the third one split off by the crystal field, as $\Gamma_{7}$ [24], using the notations of Ref. [25]. The threefold valence-band splitting is inherited by the weakly bound hole, and when the symmetry is lowered to $\overline{\mathrm{C}}_{3 v}$ the above representations are compatible with the following representations of $\overline{\mathbf{C}}_{3 v}: \Gamma_{7}\left(\overline{\mathbf{C}}_{6 v}\right) \rightarrow \Gamma_{4}\left(\overline{\mathbf{C}}_{3 v}\right)$, and $\Gamma_{9}\left(\overline{\mathbf{C}}_{6 v}\right) \rightarrow \Gamma_{5}+\Gamma_{6}=\Gamma_{56}\left(\overline{\mathbf{C}}_{3 v}\right)$. [In the last relation we use the notation $\Gamma_{56}$ for the direct sum of the representations $\Gamma_{5}+\Gamma_{6}$, which are degenerate by time reversal (Kramers) degeneracy.] The resulting symmetry of the bound-exciton states is described by representations which are a direct product of the representation for $\mathrm{Nb}^{-}$(fully symmetric, $\Gamma_{1}$, or $A_{1}$ ) and one of the representations for the hole $\left(\Gamma_{4}\right.$ or $\left.\Gamma_{56}\right)$, i.e., just the same as that of the hole alone. At this point we do not know which of the three possible hole (and therefore exciton) states is lowest in energy (one of the two $\Gamma_{4}$ states or the $\Gamma_{56}$ state), but later we will see that the actual order is $\Gamma_{4}, \Gamma_{56}$, and $\Gamma_{4}$ in order of ascending energies, with the former two states split by spin-orbit interaction, as depicted schematically in Fig. 3(b). (Thus, the ordering of the two lowest-energy states is reversed compared to the ordering in the valence band.) Assuming that these three states are close enough in order to be populated already at rather low temperatures, transitions involving either of them as initial state in the exciton recombination are expected.

The final state of the transition is just the neutral niobium center $\mathrm{Nb}^{0}$, which will be treated for the moment as possessing $\mathrm{C}_{3 v}$ symmetry with an $e$ orbital occupied by three electrons (or, alternatively, one hole) [16]. The orbital angular momentum associated with an $e$ state will couple to the spin of the hole, which results in spin-orbit splitting into $\Gamma_{4}$ and $\Gamma_{56}$ states (analogous to the spin-orbit splitting for a weakly bound hole just considered). However, we will show that the splitting for a tightly bound hole can be assumed much smaller than that for a free (or weakly bound) hole and it may well be unresolvable in our experiments, i.e., within the line width of the observed PL lines, so that the ground state behaves as a single level. Assuming the level scheme discussed above 
and presented in Fig. 3(b), it is straightforward to deduce the selection rules for optical transitions within $\mathrm{C}_{3 v}$ between the excited- and ground-state manifolds. The allowed transitions and their polarizations are depicted by the vertical arrows in Fig. 3(b). In comparing the selection rules to the lower-energy three lines in the spectrum (cf. the polarization of $\mathrm{Nb}_{0}, \mathrm{Nb}_{1}$, and $\mathrm{Nb}_{2}$ in Fig. 2) we notice that the transition $\Gamma_{56} \rightarrow \Gamma_{56}$ associated with $\mathrm{Nb}_{1}$ polarized parallel to the $c$ axis $(\mathbf{E} \| \mathbf{c})$ is strong, but the transition between $\Gamma_{4}$ and $\Gamma_{56}$ states which are allowed in $\mathbf{E} \perp \mathbf{c}$ polarization are either very weak, or not observed at all, because these transitions require spin flip.

Thus, assuming negligible spin-orbit splitting of the ground state within $\mathrm{C}_{3 v}$ symmetry, three transitions corresponding to the three closely spaced hole (and exciton) initial states are expected and the selection rules for the spin-flip-free transitions dictate that $\Gamma_{4} \rightarrow \Gamma_{4}$ is polarized both parallel and perpendicular to the $c$ axis $(\mathbf{E} \| \mathbf{c}$ and $\mathbf{E} \perp \mathbf{c})$, whereas the $\Gamma_{56} \rightarrow \Gamma_{56}$ transition is allowed only with $\mathbf{E} \| \mathbf{c}$ polarization. The former corresponds to the polarization of $\mathrm{Nb}_{0}$ and $\mathrm{Nb}_{2}$ and the latter to that of $\mathrm{Nb}_{1}$. Consequently, the energy-level scheme and selection rules suggested in Fig. 3(b) are in very good agreement with the experimental data presented so far. The possible origin of the remaining $\mathrm{Nb}_{3}, \mathrm{Nb}_{4}$, and $\mathrm{Nb}_{5}$ lines will be discussed later.

\section{Results from first-principles calculations for the Nb-bound exciton}

The energy levels associated with $\mathrm{Nb}^{0}, \mathrm{Nb}^{+}$, and $\mathrm{Nb}^{-}$ have been studied from first principles earlier [16]. These results are used in Fig. 3(a). The method employed in [16] is based on the HSE06 range-separated functional, which is capable of describing accurately both the host material (i.e., reproducing correctly the band gap and bulk properties), as well as excitation energies using constrained occupation technique [26]. Nevertheless, our recent study on the accuracy of the HSE06 functional on systems containing strongly correlated states (e.g., due to transition metal impurities), and noncorrelated semiconductor host states revealed that HSE06 may fail to accurately describe simultaneously both states in some cases [27]. Since our purpose is estimation of the energy of the excited state of $\mathrm{Nb}$ in the ASV $h$ - $h$ configuration from first principles, the accuracy of the HSE06 functional is examined carefully in the present work. The first-principles calculations were carried out on a large, 576-atom supercell with one $\mathrm{Nb}$ atom in the ASV $h-h$ configuration. In the calculations plane wave basis set with cutoff of $420 \mathrm{eV}$ and projector-augmented-wave (PAW) potentials [28] was used as implemented in the Vienna $A b$ initio Simulation Package (VASP) code [29]. The spin-orbit splitting is not considered, therefore the valence-band edge at the $\Gamma$ point of the perfect crystal (without any defect) consists of a doubly degenerate state and a nondegenerate state at lower-energy split off by the crystal field of $4 \mathrm{H}-\mathrm{SiC}$. In a perfect supercell (without $\mathrm{Nb}$ defect) the crystal-field splitting is calculated to be $\sim 45 \mathrm{meV}$.

The check of the applicability of the HSE06 functional to the case of $\mathrm{Nb}$ will be outlined shortly here. In the case of the exact functional the relation between the Kohn-Sham (KS) eigenvalues and the total energy differences fulfills the so-called generalized Koopmans' condition [30,31], i.e., the
$\mathrm{KS}$ eigenvalues are unchanged upon the occupation of the corresponding orbital and the increment of the total energy is equal to the KS eigenvalue. We tested the fulfillment of this condition in a similar way as in our previous work [27]. In order to avoid the effect of the geometry relaxation on the eigenvalues and total energies we have fixed the geometry to that in the negatively charged state $\mathrm{Nb}^{-}$, i.e., $\mathrm{C}_{3 v}$, where the double-degenerate $e$ state is fully occupied. To obtain all the quantities needed for the examination we performed calculations for the neutral and negatively charged states on the fixed geometry. In the latter state the defect electrostatically interacts with itself and with the neutralizing "jellium" background that shifts the total and KS energies. Due to the application of large supercell (576 atoms), this effect is reduced, but still of the order of $0.1 \mathrm{eV}$. This remaining energy shift can be removed from the total energy by the charge correction scheme of Freysoldt et al. [32]. For the KS energies there is no such thoroughly tested method; therefore, in order to avoid the uncertainty of the $\varepsilon_{K S}^{-, o c c}$ in the negatively charged state we used $\varepsilon_{K S}^{0, \text { unocc }}$ in the examination of the condition of exactness;

$$
\varepsilon_{K S}^{0, \text { unocc }} \approx\left(E^{-}+\Delta E_{c c}^{-}\right)-E^{0},
$$

where $E^{0}$ and $E^{-}$are the total energies in neutral and negative charge state, respectively, $\Delta E_{c c}^{-}$is the charge correction, $\varepsilon_{K S}^{-, o c c}$ is the energy of the highest occupied KS orbital in the negative charge state $\left(\mathrm{Nb}^{-}\right)$, and $\varepsilon_{K S}^{0, \text { unocc }}$ is the energy of the lowest unoccupied $\mathrm{KS}$ orbital in the neutral charge state $\left(\mathrm{Nb}^{0}\right)$. The above equality is satisfied within a margin of $0.02 \mathrm{eV}$, therefore we conclude that the excitation energies can be calculated within the HSE06 method with reasonable accuracy.

As already discussed in Sec. IV A, the energy of the exciton bound to $\mathrm{Nb}$ can be estimated by considering the weakly bound hole as virtually free, i.e., residing at the top of the valence band. Using first-principles calculation, however, makes possible estimation of the exciton binding energy when the hole is in each of the bands related to the valence-band top, the uppermost (double-degenerate) band, or the higherin-energy crystal-field split-off band. The energy difference between these two configurations will give us an estimate for the crystal-field splitting of the hole involved in the Nb-bound exciton, from first principles.

The calculated excitation energies are $1.4204 \mathrm{eV}$ when the hole is at the highest double-degenerate valence-band edge, and $1.4458 \mathrm{eV}$ when the hole is at the nondegenerate valence-band edge. Thus, the calculation predicts a reduction of the crystal-field splitting from $\sim 45 \mathrm{meV}$ (in perfect crystal) to $\sim 25 \mathrm{meV}$ in the states of the $\mathrm{Nb}$-bound exciton. This value is probably not very accurate being smaller than the accuracy of the calculation $(\sim 0.1 \mathrm{eV})$, but the trend of significant quenching of the crystal-field splitting for the weakly bound hole is in good agreement with the experimental data and justifies the assignment made earlier for the triplet $\mathrm{Nb}_{0}-\mathrm{Nb}_{2}$ to spin-orbit and crystal-field splitting of the excited state. The calculated energy stored in the exciton $(\sim 1.4 \mathrm{eV})$ and released as a photon observed in PL is also in very good agreement with both the experimental value $(1.3825 \mathrm{eV})$ and the rough energy estimates made in Sec. IV A. 


\section{Lifetime of the Nb-related emission}

Time-resolved measurements at $2 \mathrm{~K}$ yield a lifetime of $180 \mu$ s for the $\mathrm{Nb}_{0}$ line in $4 \mathrm{H}-\mathrm{SiC}$. At higher temperatures the contributions from the individual components cannot be resolved, but the total lifetime of the Nb-related emission decreases to about $120 \mu \mathrm{s}$ at $140 \mathrm{~K}$. These relatively long lifetimes of the optical transitions indicate the absence of a concurrent nonradiative recombination channel (e.g., Auger recombination), in agreement with the proposed model. Indeed, an Auger process would require that the energy stored in the exciton $(\sim 1.4 \mathrm{eV})$ is transferred to another electron within the $\mathrm{Nb}$ center exciting it into the conduction band. Considering the energy diagram in Fig. 3(a), however, it is clear that such process would require more energy than that stored in the exciton, hence Auger recombination can be ruled out as a possible recombination mechanism.

\section{E. Origin of the three high-energy lines $\mathrm{Nb}_{3}-\mathrm{Nb}_{5}$}

Let us consider now in more detail the configurations of the ground and the excited states in connection with the appearance of the three high-energy lines $\left(\mathrm{Nb}_{3}, \mathrm{Nb}_{4}\right.$, and $\left.\mathrm{Nb}_{5}\right)$ at higher temperatures. The ground state $\left(\mathrm{Nb}^{0}\right)$ has $\mathrm{C}_{1 h}$ symmetry [16], whereas the excited state $\left(\mathrm{Nb}^{-}\right.$plus weakly bound hole) is properly described by $\mathrm{C}_{3 v}$. The PLE results suggest that transitions from the ground state of $\mathrm{C}_{1 h}$ symmetry to the excited state of $\mathrm{C}_{3 v}$ symmetry (and vice versa in PL at higher temperatures) are possible, which at first glance is a violation of the Franck-Condon principle, because the "relaxed" position of the $\mathrm{Nb}$ atom changes during the transition.

In this section we provide a tentative qualitative explanation for the appearance of the three high-energy lines in PL and PLE. The results obtained from first principles suggest that the change in the $\mathrm{Nb}$ configuration is extremely small. When the charge state changes from $\mathrm{Nb}^{-}\left(\mathrm{C}_{3 v}\right)$ to $\mathrm{Nb}^{0}\left(\mathrm{C}_{1 h}\right)$, the calculation yields that the relaxed position in $\mathrm{C}_{1 h}$ is only $\sim 0.02 \AA$ away from the $\mathrm{C}_{3 v}$ symmetry axis, accompanied by larger (but still very small) relaxation of $\sim 0.06 \AA$ along the $C_{3 v}$ axis towards the three nearest carbon atoms due to weakening of one bond to one of the three nearest silicon atoms. While the average "relaxed" position of $\mathrm{Nb}^{0}$ is in $\mathrm{C}_{1 h}$ symmetry, it may be speculated that the configuration resumes dynamically $\mathrm{C}_{3 v}$ symmetry due to zero-point vibrations, in analogy with the dynamic Jahn-Teller effect, if we admit that the change in the configuration of $\mathrm{Nb}$ between the two charge states is of the order of the zero-point vibration amplitudes for $\mathrm{Nb}^{0}$. Thus, one can assume finite probability for finding $\mathrm{Nb}^{0}$ in the $\mathrm{C}_{3 v}$ symmetry inherent to the excited state (i.e., to $\mathrm{Nb}^{-}$) and direct excitation in accord with the Franck-Condon principle becomes possible. This provides reasonable explanation for the observation of the $\mathrm{Nb}_{2}$ line in the PLE spectrum. On the other hand, the existence of $\mathrm{Nb}^{0}$ (the ground state) in $\mathrm{C}_{1 h}$ symmetry provides us with a reason to expect an excited state associated with this configuration, i.e., when the hole is bound to $\mathrm{Nb}^{-}$in $\mathrm{C}_{1 h}$ symmetry. This state of $\mathrm{C}_{1 h}$ symmetry for the bound exciton is not the lowest energy state; the latter is achieved after relaxation to $\mathrm{C}_{3 v}$ symmetry. In addition, if this state exists it will exhibit the threefold splitting stemming from the valence-band splitting, in analogy with the previously considered $\mathrm{C}_{3 v}$ configuration. Both symmetries for the bound exciton can again be considered as connected owing to the zero-point vibrations of the system. It should be noted, however, that due to the stronger binding to the ligands the average zero-point vibration amplitude for $\mathrm{Nb}^{-}$is expected to be smaller than that for $\mathrm{Nb}^{0}$, which might explain why elevated temperature is needed to activate the observation of the higher-energy triplet in PL. We note here that we conducted calculations from first principles, when the excited state was "forced" initially from the $\mathrm{C}_{1 h}$ configuration inherent to the ground state, and the system was left to relax to a state of minimum energy, which might be the sought $\mathrm{C}_{1 h}$ state. Due to limited accuracy, however, this calculation could neither confirm nor disprove the existence of this state.

Thus, our tentative explanation of the appearance of the three higher-energy lines is based on the assumption that the bound exciton has an excited state of $\mathrm{C}_{1 h}$ symmetry, which exhibits thermalization (becomes populated) with increasing temperatures. This notion is in agreement with the PLE results, for direct excitation from the ground state of $\mathrm{C}_{1 h}$ symmetry of $\mathrm{Nb}_{0}$ into this state is possible at any low temperature. This notion is consistent also with the very long (compared to other centers) lifetimes of the optical transitions. Furthermore, in view of the small deviation from $\mathrm{C}_{3 v}$ symmetry we expect the optical properties of the high-energy lines $\left(\mathrm{Nb}_{3}-\mathrm{Nb}_{5}\right)$ in terms of polarization and Zeeman splitting to be similar to the properties of the low-energy triplet $\left(\mathrm{Nb}_{0}-\mathrm{Nb}_{2}\right)$ because the descent to $\mathrm{C}_{1 h}$ symmetry can be viewed as small perturbation which does not affect strongly the selection rules considered in $\mathrm{C}_{3 v}$ symmetry. Indeed, the middle line in the high-energy triplet $\mathrm{Nb}_{4}$ exhibits $\mathbf{E} \| \mathbf{c}$ polarization in both $4 \mathrm{H}$ and $6 \mathrm{H} \mathrm{SiC}$, similar to its analog $\mathrm{Nb}_{1}$ line in the low-energy triplet. The remaining two lines $\left(\mathrm{Nb}_{3}\right.$ and $\mathrm{Nb}_{5}$, whenever observable) do not show preferable polarization in analogy with the corresponding $\mathrm{Nb}_{0}$ and $\mathrm{Nb}_{2}$ lines (cf. Fig. 2). Finally, the Zeeman splitting of the $\mathrm{Nb}_{3}$ and $\mathrm{Nb}_{5}$ lines which can be observed for magnetic field parallel to the $c$ axis shows essentially the same pattern as that for the $\mathrm{Nb}_{0}$ and $\mathrm{Nb}_{2}$ lines, as will be demonstrated in Sec. V. Thus, the notion of an excited state of the exciton of $\mathrm{C}_{1 h}$ symmetry associated with the JahnTeller distortion of $\mathrm{Nb}^{0}$ is in agreement with all experimental observations, but its existence remains arguable until proved or dismissed using more accurate calculation methods.

\section{ZEEMAN EFFECT: MODELS AND EXPERIMENT}

\section{A. Experimental results}

Since the behavior of the excited-state manifold under magnetic field is of interest, the Zeeman spectra were recorded not only at $2 \mathrm{~K}$ but also at somewhat elevated temperatures (5-30 K) allowing the observation of the lower-energy triplet $\mathrm{Nb}_{0}-\mathrm{Nb}_{2}$. The high-energy triplet $\mathrm{Nb}_{3}-\mathrm{Nb}_{5}$ remains too weak and temperature-associated line broadening prevents detailed study of the Zeeman effect on all the three lines; however, it was possible to record the $\mathrm{Nb}_{3}$ (and, to some extent, the $\mathrm{Nb}_{5}$ ) line behavior for a magnetic field parallel to the $c$ axis $(\mathbf{B} \| \mathbf{c})$. An applied magnetic field reduces the symmetry of a defect in different ways depending on the orientation of the field with respect to the crystallographic axes ( $c$ axis). Considering the $\mathrm{Nb}$-bound exciton of $\mathrm{C}_{3 v}$ symmetry, 


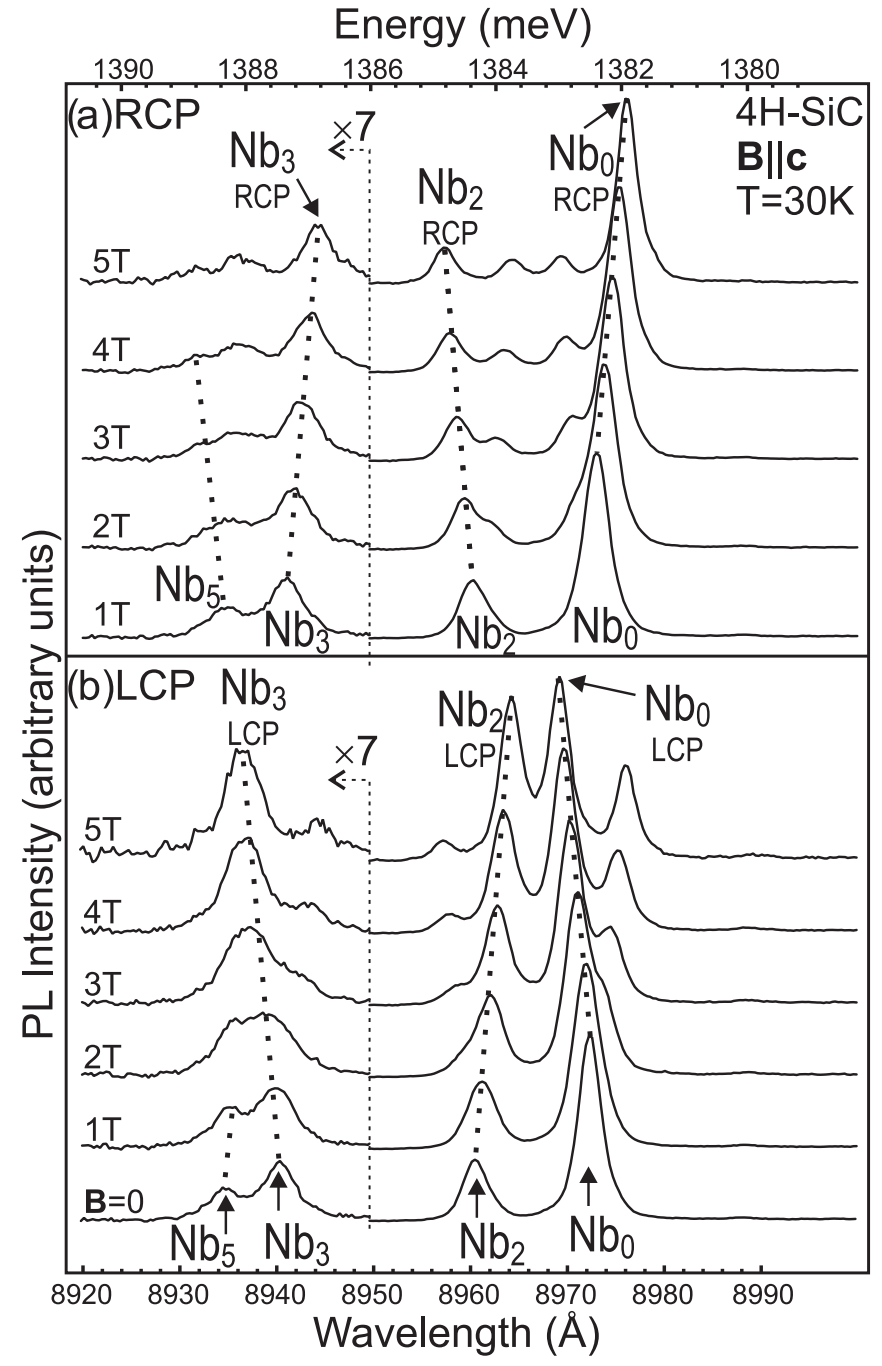

FIG. 4. Zeeman splitting of the Nb-related lines recorded with two different polarizations in magnetic field $\mathbf{B} \| \mathbf{c}$ up to $5 \mathrm{~T}$. Parts (a) and (b) of the figure refer to right- and left-hand circular polarizations, respectively. Note the scale changes in the high-energy region of the spectra. (Data is adapted with permission from Ref. [18].)

magnetic field $\mathbf{B} \| \mathbf{c}$ reduces the symmetry to $\mathrm{C}_{3}$, whereas the highest possible symmetry in a magnetic field perpendicular to the crystal $c$ axis $(\mathbf{B} \perp \mathbf{c})$ is $\mathrm{C}_{1 h}$. If simply a state with spin equal to $1 / 2$ is considered (without regard for spin-orbit interaction), one intuitively expects that magnetic field $B$ will split it into two states with energies $\pm \frac{\mu B}{2}$ with respect to the zero-field energy. Also, if a hole state is considered, the state corresponding to spin (or total angular momentum) $+1 / 2$ lies lower in energy. However, we have to deal with excited and ground states involved in the PL transition which are subject to spin-orbit interaction, in accord with our model of a Nb-bound exciton. Therefore, in the next section we will consider in detail two phenomenological models which will be used in the interpretation of the experimental data.

The spectra of $\mathrm{Nb}$-doped $4 \mathrm{H}-\mathrm{SiC}$ at $30 \mathrm{~K}$ in magnetic field B $\|$ c up to 5 T are shown in Fig. 4. Note that due to the Faraday configuration imposed by our experimental geometry the $\mathrm{Nb}_{1}$ line is not detected, because it is polarized parallel to the $c$ axis

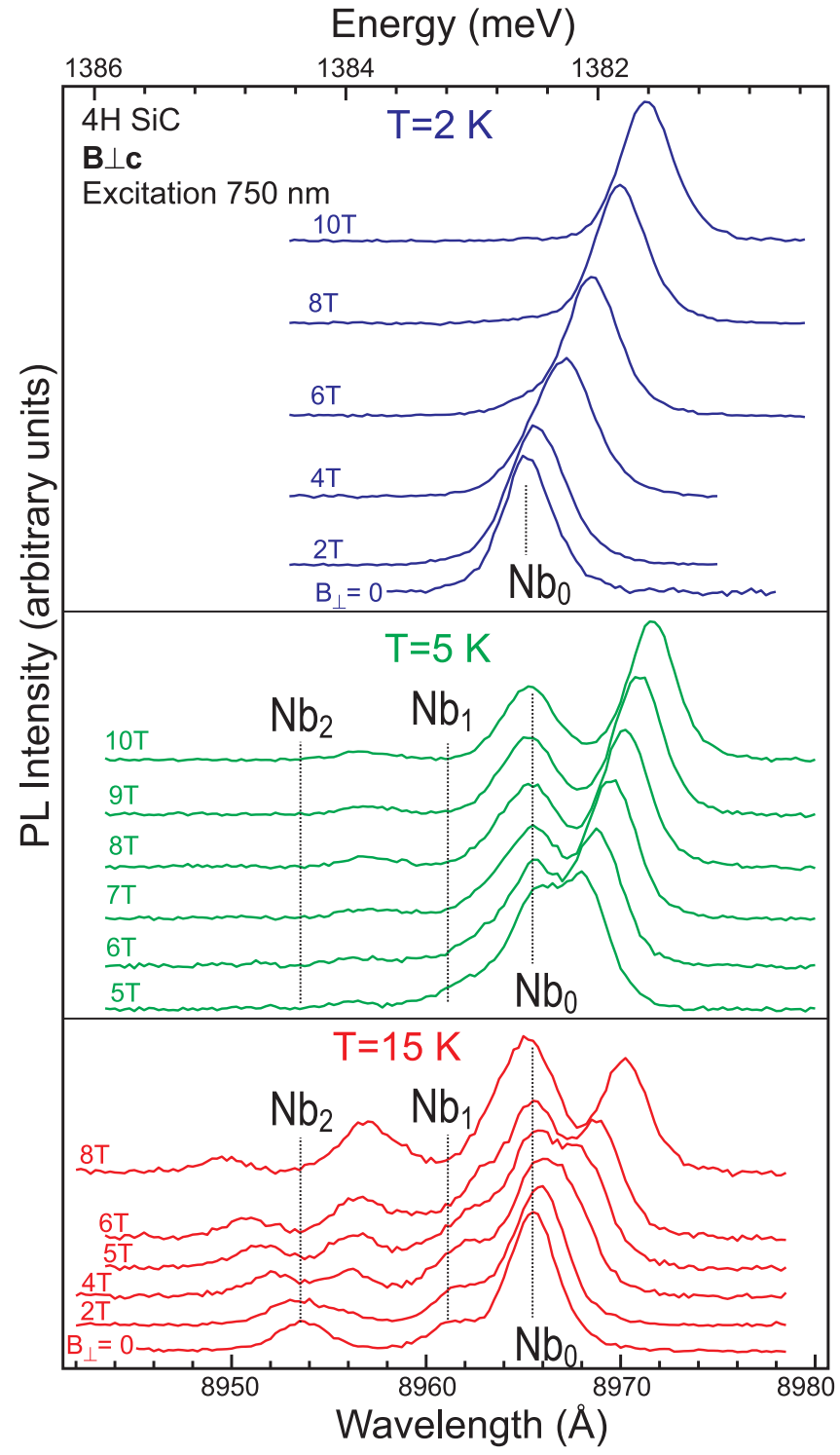

FIG. 5. (Color online) Illustration of the Zeeman splitting of the $\mathrm{Nb}$-related lines in magnetic field $\mathbf{B} \perp \mathbf{c}$ up to $10 \mathrm{~T}$ at three different temperatures as denoted in each panel. Different temperatures are used in order to distinguish high- from low-temperature lines in the spectra. The zero-field positions of the lines are denoted in each panel. (Some data is adapted with permission from Ref. [18].)

and, consequently, propagates in the basal plane. The spectra in magnetic field $\mathbf{B} \perp \mathbf{c}$ up to $10 \mathrm{~T}$ for three different temperatures are shown in Fig. 5. We notice that although in this case the light was collected through the edge of the sample enabling detection of polarization $\mathbf{E} \| \mathbf{c}$, the $\mathrm{Nb}_{1}$ line is observed weakly only in the spectra recorded at $15 \mathrm{~K}$. We attribute this weak intensity to the fact that the light is collected via fiber, so that light propagating in a spatial angle (with $\mathbf{E} \perp \mathbf{c}$ ) is collected more efficiently than light propagating in a plane (with $\mathbf{E} \| \mathbf{c}$ polarization). In addition, the distance between the fiber and the sample is uncontrollable in practice, due to shrinking of the sample holder at low temperature. Larger distance of the fiber from the edge of the sample will diminish the intensity ratio between the $\mathbf{E} \| \mathbf{c}$ and $\mathbf{E} \perp \mathbf{c}$ polarizations. 


\section{B. Phenomenological models describing the Zeeman splitting}

The interpretation of the experimental data, especially for the case $\mathbf{B} \perp \mathbf{c}$ (Fig. 5), requires consideration of the spin Hamiltonian of the excited and ground states. As will be seen, this consideration will allow us to explain the nonlinear splitting of the PL lines observed with magnetic field $\mathbf{B} \perp \mathbf{c}$. In addition, we will obtain justification for the much simpler and rather intuitive picture observed for the case $\mathbf{B} \| \mathbf{c}$, resembling the splitting of states with spin $1 / 2$ already mentioned in Sec. V A. We will also address the selection rules in magnetic field.

In building the phenomenological Hamiltonian describing the ground and excited states of the $\mathrm{Nb}$ center we have to account for the spin-orbit coupling pertinent to the hole. On one hand, we can treat the orbital angular momentum $l$ and spin $s$ of the hole separately and describe the spin-orbit coupling introducing appropriate operators in the Hamiltonian (weak to intermediate spin-orbit coupling); this model will be referred to further on as the $l s$ model. On the other hand, we may consider another phenomenological model where $l$ and $s$ are already coupled into total angular momentum $j=l+s$, which behaves as a single quantity in external magnetic field (strong spin-orbit coupling); this model will be referred to as the $j$ model. In the following we shall work out both models and compare their predictions with the experimental data. It should be noticed in advance, however, that there is no reason to expect that the same $(j$ or $l s)$ model provides a good description of both the excited and the ground states. This will be stipulated further during the comparison with the experimental data.

Let us consider now the $l s$ model. Disregarding at first the spin of the free (or weakly bound) hole, we notice that its wave function (the orbital part) behaves as one of the components of a $p$-like state (in the common $s, p, d, f, \ldots$ notation familiar from the hydrogen atom). In $T_{d}$ (or spherical) symmetry a $p$ like state is triply degenerate, i.e., the three components $p_{x}, p_{y}$, and $p_{z}$ have the same energy. Consequently, we may represent these three wave functions corresponding to orbital angular momentum $l=1$ with the combinations $\left|l, m_{l}\right\rangle$, where $m_{l}=$ $0, \pm 1$ is the orbital angular momentum quantum number. In uniaxial symmetry $\left(\mathrm{C}_{6 v}\right.$ for a free hole, or $\mathrm{C}_{3 v}$ for the weakly bound one) with the $z$ axis along the symmetry $c$ axis, the $p_{x}$ and $p_{y}$ components are still degenerate, but the energy of the $p_{z}$ counterpart will be different due to the crystal-field splitting. The corresponding wave functions within the $\left|l, m_{l}\right\rangle$ notation can be denoted as $|1,+1\rangle,|1,-1\rangle$ for the degenerate $p_{x}, p_{y}$ components, and $|1,0\rangle$ for the $p_{z}$ component. The notation can be simplified by omitting the index $l=1$, thus the three functions above can be denoted simply as $\left|m_{l}\right\rangle$, i.e., $|1\rangle,|-1\rangle$, and $|0\rangle$. This results in the following Hamiltonian with account for crystal-field splitting (but without account for spin):

$$
\hat{H}_{0}=|0\rangle\langle 0|+\varepsilon_{c f}(|1\rangle\langle 1|+|-1\rangle\langle-1|),
$$

where $\varepsilon_{c f}<0$ is the energy associated with the crystal field. Its value is negative, because we describe a $p$-like hole, for which the double-degenerate state (without account for spin-orbit coupling) lies lower in energy than the nondegenerate $|0\rangle$ state. The wave function $|0\rangle$ transforms as the fully symmetric $\Gamma_{1}$ representation of $\mathrm{C}_{3 v}$, whereas the two functions $|1\rangle$ and $|-1\rangle$ form the basis for the two-dimensional $\Gamma_{3}$ (denoted sometimes $E$ ) representation of $\mathrm{C}_{3 v}$.

Account for spin doubles the degeneracy of the three considered states. The wave functions can be denoted now with $\left|m_{l}, m_{s}\right\rangle$, where $m_{s}= \pm 1 / 2$ is the spin quantum number. Also, the spin-orbit interaction becomes active, which is accounted for by the spin-orbit Hamiltonian

$$
\hat{H}_{S O}=\lambda_{\|} \hat{l}_{z} \hat{s}_{z}+\lambda_{\perp}\left(\hat{l}_{+} \hat{s}_{-}+\hat{l}_{-} \hat{s}_{+}\right) .
$$

Here, $\lambda_{\|}$and $\lambda_{\perp}$ are phenomenological constants with dimension of energy determining the strength of the spin-orbit interaction along and perpendicular to the crystal axis $(c$ axis), both negative because we describe a hole; $\hat{l}_{ \pm}=\hat{l}_{x} \pm i \hat{l}_{y}$, $\hat{s}_{ \pm}=\hat{s}_{x} \pm i \hat{s}_{y}$, and $\hat{l}_{x}, \hat{l}_{y}, \hat{l}_{z}\left(\hat{s}_{x}, \hat{s}_{y}, \hat{s}_{z}\right)$ are the components of the orbital angular momentum (spin) operator, respectively. The symmetry of the wave functions $\left|m_{l}, m_{s}\right\rangle$ is the following. Each of the two pairs $\{|0,1 / 2\rangle,|0,-1 / 2\rangle\}$ and $\{|-1,-1 / 2\rangle,|1,1 / 2\rangle\}$ forms a basis for the two-dimensional $\Gamma_{4}$ representation of $\overline{\mathrm{C}}_{3 v}$, whereas the two remaining functions $|-1,-1 / 2\rangle$ and $|1,1 / 2\rangle$ correspond to the $\Gamma_{56}$ representation. The Hamiltonian in absence of magnetic field then becomes $\hat{H}_{0}+\hat{H}_{S O}$.

Finally, we add the Zeeman Hamiltonian $\hat{H}_{Z}$, which in our case of $p$-like hole in magnetic field $\mathbf{B}$ with components $B_{x}, B_{y}, B_{z}$ takes the form

$$
\hat{H}_{Z}=-\mu(\hat{l}+2 \hat{s}) B=-\mu\left(\hat{l}_{z}+2 \hat{s}_{z}\right) B_{\|}-\frac{1}{2} \mu\left[\left(\hat{l}_{+}+2 \hat{s}_{+}\right) B_{-}+\left(\hat{l}_{-}+2 \hat{s}_{-}\right) B_{+}\right],
$$

where $B_{ \pm}=B_{x} \pm i B_{y}, B_{\|}=B_{z}, \mu$ is the Bohr magneton, and the overall minus sign in the Hamiltonian is due to the fact that we describe a hole. In the following we use also the notation $B_{\perp}=B_{+}$, hence $B_{-}=B_{\perp}^{*}$ (the asterisk superscript denotes complex conjugation).

We now expand the total Hamiltonian $\hat{H}=\hat{H}_{0}+\hat{H}_{S O}+\hat{H}_{Z}$ in the basis of the six wave functions $|1\rangle=|0,1 / 2\rangle,|2\rangle=$ $|0,-1 / 2\rangle,|3\rangle=|-1,1 / 2\rangle,|4\rangle=|1,-1 / 2\rangle,|5\rangle=|-1,-1 / 2\rangle$, and $|6\rangle=|1,1 / 2\rangle$ and obtain the general form of the Hamiltonian matrix (in general magnetic field)

$$
H=E I+\left(\begin{array}{cccccc}
-\mu B_{\|} & -\mu B_{\perp}^{*} & -\frac{\mu B_{\perp}^{*}}{\sqrt{2}} & \frac{\lambda_{\perp}}{\sqrt{2}} & 0 & -\frac{\mu B_{\perp}}{\sqrt{2}} \\
-\mu B_{\perp} & \mu B_{\|} & \frac{\lambda_{\perp}}{\sqrt{2}} & -\frac{\mu B_{\perp}}{\sqrt{2}} & -\frac{\mu B_{\perp}^{*}}{\sqrt{2}} & 0 \\
-\frac{\mu B_{\perp}}{\sqrt{2}} & \frac{\lambda_{\perp}}{\sqrt{2}} & \varepsilon_{c f}-\frac{\lambda_{\|}}{2} & 0 & -\mu B_{\perp}^{*} & 0 \\
\frac{\lambda_{\perp}}{\sqrt{2}} & -\frac{\mu B_{\perp}^{*}}{\sqrt{2}} & 0 & \varepsilon_{c f}-\frac{\lambda_{\|}}{2} & 0 & -\mu B_{\perp} \\
0 & -\frac{\mu B_{\perp}}{\sqrt{2}} & -\mu B_{\perp} & 0 & \varepsilon_{c f}+\frac{\lambda_{\|}}{2}+2 \mu B_{\|} & 0 \\
-\frac{\mu B_{\perp}^{*}}{\sqrt{2}} & 0 & 0 & -\mu B_{\perp}^{*} & 0 & \varepsilon_{c f}+\frac{\lambda_{\|}}{2}-2 \mu B_{\|}
\end{array}\right) .
$$


Here $I$ is the unit matrix and $E$ is simply a constant energy shift (of the order of the energies of the PL transitions) applied to the eigenenergies of Eq. (5) in order to reproduce the shift of the excited states from the ground state at zero magnetic field. The average energy of the ground state at zero field will be chosen as reference (zero) energy.

We see from Eq. (5), e.g., by considering the form of the matrix at zero magnetic field $(\mathbf{B}=0)$ that $\lambda_{\perp}$ is responsible for mixing of the two doublets of $\Gamma_{4}$ symmetry, whereas $\lambda_{\|}$is responsible for the splitting of the $\Gamma_{56}$ state from the nearest in energy $\Gamma_{4}$ state. Furthermore, at zero magnetic field all eigenvalues are doubly degenerate and can be presented analytically:

$$
\begin{aligned}
\varepsilon_{1,3} & =\frac{1}{2}\left(\varepsilon_{c f}-\frac{\lambda_{\|}}{2}\right)\left[1 \mp \sqrt{1+2\left(\frac{\lambda_{\perp}}{\varepsilon_{c f}-\lambda_{\|} / 2}\right)^{2}}\right], \\
\varepsilon_{2} & =\varepsilon_{c f}+\frac{\lambda_{\|}}{2} .
\end{aligned}
$$

The "-" sign in Eq. (6a) refers to $\varepsilon_{1}$, the "+" sign to $\varepsilon_{3}$, and $\varepsilon_{1}<\varepsilon_{2}<\varepsilon_{3}$ (recall that $\lambda_{\|}<0$ ). The differences between the states should be compared to the zero-field splitting of the observed PL lines, i.e., $\varepsilon_{3}-\varepsilon_{1}=1.8 \mathrm{meV}$, and $\varepsilon_{2}-\varepsilon_{1}=$ $0.65 \mathrm{meV}$ (cf. Table I). This yields the equations

$$
\begin{array}{r}
\sqrt{\left(\varepsilon_{c f}-\frac{\lambda_{\|}}{2}\right)^{2}+2 \lambda_{\perp}^{2}}=1.8 \mathrm{meV} \text { and } \\
\frac{1}{2} \varepsilon_{c f}+\frac{3}{4} \lambda_{\|}=-0.25 \mathrm{meV} .
\end{array}
$$

The first equation in (7) shows that consistency with the experimental data requires $\left|\lambda_{\perp}\right|<1.8 / \sqrt{2} \mathrm{meV} \approx 1.273 \mathrm{meV}$. The value of $\left|\lambda_{\perp}\right|$ is also bound from below, $\left|\lambda_{\perp}\right|>1.23 \mathrm{meV}$ (approximately), in order to ensure the ordering $\varepsilon_{1}<\varepsilon_{2}<$ $\varepsilon_{3}$, which is in agreement with the experimental data (the middle line in the low-energy PL triplet has $\mathbf{E} \| \mathbf{c}$ polarization stemming from $\Gamma_{56} \rightarrow \Gamma_{56}$ transition). Once $\lambda_{\perp}$ is chosen, the values for $\varepsilon_{c f}$ and $\lambda_{\|}$, which ensure the same splitting as the experimental one, are determined by solving Eqs. (7). We will see later that the choice of larger (by absolute value) $\lambda_{\perp}$ leads to similar splitting of the two $\Gamma_{4}$ states in magnetic field $\mathbf{B} \| \mathbf{c}$, which is consistent with the experimental data. Using, for instance, $\lambda_{\perp}=-1.270 \mathrm{meV}$ (close to the maximum absolute value), we obtain $\lambda_{\|} \approx-0.190 \mathrm{meV}$ and $\varepsilon_{c f} \approx-0.214 \mathrm{meV}$ from Eqs. (7). The choice of the energy shift $E=1383.46 \mathrm{meV}$ in Eq. (5) puts the lowest-energy PL transition at the same energy as the experimental one $(\sim 1382.5 \mathrm{meV}$ in $4 \mathrm{H} \mathrm{SiC}$; cf. Table I). The corresponding dependence of the excited states on the magnetic field is depicted in Figs. 6(b) and 6(d) for $\mathbf{B} \| \mathbf{c}$ and $\mathbf{B} \perp \mathbf{c}$, respectively.

The ground state of $\mathrm{Nb}^{0}$, represented by a hole in $e$ state in $\mathrm{C}_{3 v}$ symmetry effective after the exciton recombination and before relaxation to $\mathrm{C}_{1 h}$ symmetry, could be treated in a similar way within the $l s$ model. Although no relation to the valence-band states is expected because the hole is tightly bound in this state, a state of $e$ symmetry $\left(\Gamma_{3}\right.$ in $\left.\mathrm{C}_{3 v}\right)$ can still be roughly approximated as stemming from degenerated $p_{x}$ and $p_{y}$ orbitals of a $p$-like state, with the corresponding $p_{z}$ orbital being remote in energy and, therefore, not participating
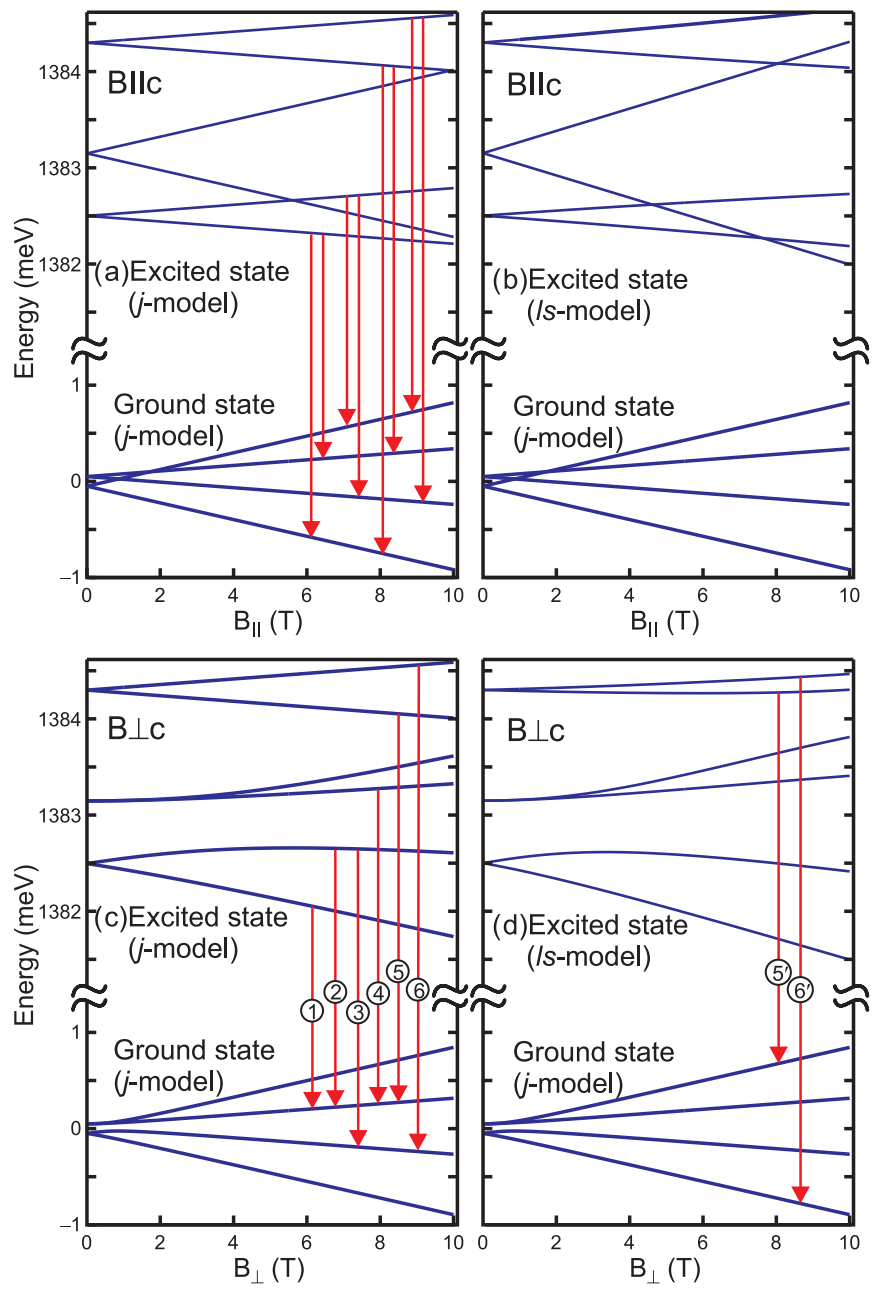

FIG. 6. (Color online) Splitting of the energy levels in magnetic field $\mathbf{B} \| \mathbf{c}$ [panels (a) and (b)] and $\mathbf{B} \perp \mathbf{c}$ [panels (c) and (d)] calculated using the $j$ model [panels (a) and (c)] and the $l s$ model [panels (b) and (d)] for the excited states, and the $j$ model for the ground state with small arbitrary splitting of $0.1 \mathrm{meV}$. Note that all transitions denoted with arrows in part (a) occur at energy shifts very close to $\pm \mu B_{\|}$from the zero-field line position (exactly $\pm \mu B_{\|}$if the ground-state zero-field splitting is zero). For the case $\mathbf{B} \perp \mathbf{c}$, only transitions used in Fig. 7 are denoted and enumerated to comply with the discussion in text.

in the resulting Hamiltonian. The Hamiltonian built according to this concept would resemble the lower right $4 \times 4$ minor of the matrix in Eq. (5), but of course with different values for the parameters $\varepsilon_{c f}$ and $\lambda_{\|}$. However, the set of basis functions would now involve only four functions: the pair $|-1,1 / 2\rangle$, $|1,-1 / 2\rangle$ transforming as $\Gamma_{4}$, and the pair $|-1,-1 / 2\rangle,|1,1 / 2\rangle$ transforming as $\Gamma_{56}$. Neglecting the second $\Gamma_{4}$ state will neglect also the coupling of the two $\Gamma_{4}$ states (equivalent to $\lambda_{\perp}=0$ ), and it will be shown shortly that we have to resort to the $j$ model in the description of the ground state. It should be noted here, that the considered model of a tightly bound hole for the ground state is consistent with the large spin-orbit splitting usually associated with transition metals. Indeed, although the treatment of hole states as $p$ like would only be a rough approximation for the ground state, the $l s$ model shows that a large splitting of one of the $p$-like 
components (transforming as $\Gamma_{4}$ ) can be achieved on account of a large value of either $\varepsilon_{c f}$, or $\lambda_{\perp}$ alone, as seen for instance from Eqs. (6). For example, for $\varepsilon_{c f} \gg\left|\lambda_{\perp}\right|$, we obtain from Eqs. (6) that $\varepsilon_{1} \approx 0$ and $\varepsilon_{2,3} \approx \varepsilon_{c f} \pm \frac{\lambda_{\|}}{2}$, so that essentially the splitting between the $\Gamma_{56}$ and the higher-in-energy $\Gamma_{4}$ states is determined by $\lambda_{\|}$and may be negligibly small. The small value of $\lambda_{\|}$cannot be justified from the model, but is inferred from the experimental data showing that the ground state behaves as a single state. Consequently, its splitting (if any) is below the visible linewidth of the PL lines $(\sim 0.3 \mathrm{meV})$. Thus, when considering the $j$ model we will set the splitting for the ground state of the hole with $e$ symmetry in $\mathrm{C}_{3 v}$ to $0.1 \mathrm{meV}$, quite arbitrarily, merely with the purpose to illustrate the effect of a small splitting on the energy-level dependencies in magnetic field.

Let us now outline briefly the $j$ model, which allows us to examine a scenario when the spin and orbital angular momentum are already coupled (strongly) into a total angular momentum $j$. This case can be examined on the grounds of standard perturbation theory. We consider first the excited state. The possible values of $j$ are $1 / 2$ ( $l$ and $s$-antiparallel) and $3 / 2$ ( $l$ and $s$-parallel). Using the notation $\left|j, m_{j}\right\rangle$, where $m_{j}$ is the projection of $j$, the six wave functions

$$
H^{\prime}=\left(\begin{array}{ccc}
E_{1}-\frac{\mu B_{\|}}{2} & -\frac{\mu B_{\perp}^{*}}{2} & 0 \\
\frac{-\mu B_{\perp}}{2} & E_{1}+\frac{\mu B_{\|}}{2} & 0 \\
0 & 0 & E_{2}+\frac{\mu B_{\|}}{2} \\
0 & 0 & -\mu B_{\perp}^{*} \\
0 & 0 & -\frac{\sqrt{3} \mu B_{\perp}}{2} \\
0 & 0 & 0
\end{array}\right.
$$

Note that within the $j$ model there are no adjustable parameters. The three energy shifts corresponding to the three PL lines observed at zero field are $E_{1}=1384.3 \mathrm{meV}, E_{2}=$ $1382.5 \mathrm{meV}$, and $E_{3}=1383.15 \mathrm{meV}$, in order to comply with the experimentally observed zero-field splitting. Arguments analogical to those supplied in the consideration of the ground state within the $l s$ model suggest that the ground state can be described by a Hamiltonian resembling the lower right-hand $4 \times 4$ minor of the Hamiltonian matrix of Eq. (9), but with different values of the zero-field energies $E_{2}^{0}=-0.05 \mathrm{meV}$ and $E_{3}^{0}=0.05 \mathrm{meV}$. The superscript " 0 " denotes the energies of the ground state, and the average value of the energy of the ground state at zero field is chosen as zero-energy reference, $\left(E_{2}^{0}+E_{3}^{0}\right) / 2=0$, as noted earlier. Similar to the discussion within the $l s$ model, only four wave functions are involved in the description of the ground state, namely, $|3\rangle^{0}=$ $|3 / 2,-1 / 2\rangle,|4\rangle^{0}=|3 / 2,1 / 2\rangle,|5\rangle^{0}=|3 / 2,-3 / 2\rangle$, and $|6\rangle^{0}=$ $|3 / 2,3 / 2\rangle$, using as before the notation $\left|j, m_{j}\right\rangle$. It should be recognized that the $j$ model is free from the problem with neglect of the interaction between the two $\Gamma_{4}$ states, which was discussed when investigating the possibility to build the ground-state Hamiltonian using the $l s$ model. This is due to the fact that the spin-orbit interaction is already built into the $j$ model on a phenomenological basis. Note the arbitrary splitting of $0.1 \mathrm{meV}$ between the two components of the ground state at zero field, which was discussed earlier and involved in the description of the excited state may be denoted as $|1\rangle=|1 / 2,1 / 2\rangle, \quad|2\rangle=|1 / 2,-1 / 2\rangle, \quad|3\rangle=|3 / 2,-1 / 2\rangle$, $|4\rangle=|3 / 2,1 / 2\rangle,|5\rangle=|3 / 2,-3 / 2\rangle$, and $|6\rangle=|3 / 2,3 / 2\rangle$. As in the $l s$ model, each of the first two pairs (i.e., $\{|1\rangle,|2\rangle\}$ and $\{|3\rangle,|4\rangle\}$ with $m_{j}= \pm 1 / 2$ forms the basis for the $\Gamma_{4}$ representation, whereas the last pair transforms as the $\Gamma_{56}$ representation. The six wave functions are now assumed to be eigenfunctions of the phenomenological zero-field Hamiltonian $\hat{H}_{0}^{\prime}$ with the spin-orbit interaction and crystalfield splitting already included, which is considered as the known unperturbed Hamiltonian. Thus, we already know the zero-field splitting between the eigenenergies corresponding to the three pairs $\{|1\rangle,|2\rangle\},\{|3\rangle,|4\rangle\}$, and $\{|6\rangle,|6\rangle\}$ from the experimental energy positions of the PL lines, assuming negligible splitting in the ground state. The perturbation in magnetic field is given by the Zeeman Hamiltonian $\hat{H}_{Z}^{\prime}$, which now (within the $j$ model) reads

$$
\hat{H}_{Z}^{\prime}=-\mu \hat{j} B=-\mu \hat{j}_{z} B_{\|}-\frac{1}{2} \mu\left[\hat{j}_{+} B_{-}+\hat{j}_{-} B_{+}\right] .
$$

The Hamiltonian matrix $H^{\prime}$ corresponding to the Hamiltonian in magnetic field $\hat{H}^{\prime}=\hat{H}_{0}^{\prime}+\hat{H}_{Z}^{\prime}$ reads

$$
\left.\begin{array}{ccc}
0 & 0 & 0 \\
0 & 0 & 0 \\
-\mu B_{\perp} & -\frac{\sqrt{3} \mu B_{\perp}^{*}}{2} & 0 \\
E_{2}-\frac{\mu B_{\|}}{2} & 0 & -\frac{\sqrt{3} \mu B_{\perp}}{2} \\
0 & E_{3}+\frac{3 \mu B_{\|}}{2} & 0 \\
-\frac{\sqrt{3} \mu B_{\perp}^{*}}{2} & 0 & E_{3}-\frac{3 \mu B_{\|}}{2}
\end{array}\right) .
$$

is introduced simply with the purpose of illustrating the effect of small splitting on the energy-level dependencies in magnetic field. Explicitly, the Hamiltonian matrix describing the ground state reads

$$
H^{0}=\left(\begin{array}{cccc}
E_{2}^{0}+\frac{\mu B_{\|}}{2} & -\mu B_{\perp} & -\frac{\sqrt{3} \mu B_{\perp}^{*}}{2} & 0 \\
-\mu B_{\perp}^{*} & E_{2}^{0}-\frac{\mu B_{\|}}{2} & 0 & -\frac{\sqrt{3} \mu B_{\perp}}{2} \\
-\frac{\sqrt{3} \mu B_{\perp}}{2} & 0 & E_{3}^{0}+\frac{3 \mu B_{\|}}{2} & 0 \\
0 & -\frac{\sqrt{3} \mu B_{\perp}^{*}}{2} & 0 & E_{3}^{0}-\frac{3 \mu B_{\|}}{2}
\end{array}\right) .
$$

The resulting energy dependencies of the excited states on magnetic fields $\mathbf{B} \| \mathbf{c}$ and $\mathbf{B} \perp \mathbf{c}$ are plotted in Figs. 6(a) and $6(c)$, respectively. The ground-state dependence on magnetic field is that from the $j$ model in all four panels of Fig. 6. Note that while the splitting of the levels of the excited state predicted by the $l s$ model and the $j$ model look qualitatively similar in Fig. 6, quantitatively they are very different. However, before further consideration of these models and comparison with the experiment we will address briefly the selection rules for the two cases $\mathbf{B} \| \mathbf{c}$ and $\mathbf{B} \perp \mathbf{c}$.

\section{Selection rules in magnetic field}

In magnetic field $\mathbf{B} \| \mathbf{c}$ the symmetry descends from $\mathbf{C}_{3 v}$ to $\mathrm{C}_{3}$. The $\Gamma_{4}$ and $\Gamma_{56}$ representations of $\overline{\mathrm{C}}_{3 v}$ are compatible with the following representations of $\overline{\mathrm{C}}_{3}: \Gamma_{4}\left(\overline{\mathrm{C}}_{3 v}\right) \rightarrow \Gamma_{4}+\Gamma_{5}\left(\overline{\mathrm{C}}_{3}\right)$, 
and $\Gamma_{56}\left(\overline{\mathbf{C}}_{3 v}\right) \rightarrow \Gamma_{6}+\Gamma_{6}\left(\overline{\mathbf{C}}_{3}\right)$. The dipole operator for light polarized perpendicular to the $c$ axis $(\mathbf{E} \perp \mathbf{c})$ transforms as either $\Gamma_{2}$ or $\Gamma_{3}$ representation of $\overline{\mathrm{C}}_{3}$, corresponding to left or right circular polarization ( $\mathrm{LCP}$ or $\mathrm{RCP}$ ), respectively. Consequently, transitions from $\Gamma_{4}\left(\overline{\mathrm{C}}_{3}\right)$ to $\Gamma_{5}\left(\overline{\mathrm{C}}_{3}\right)$ are allowed with emission of photons with LCP, whereas $\Gamma_{5}\left(\overline{\mathbf{C}}_{3}\right)$ to $\Gamma_{4}\left(\overline{\mathbf{C}}_{3}\right)$ transitions produce photons with RCP. We notice that the above compatibility relations mean that double-degenerate states of the hole of spin $+1 / 2$ (transforming as $\Gamma_{4}$ of $\overline{\mathrm{C}}_{3 v}$ ) split into two states in $\mathbf{B} \| \mathbf{c}$ : the state with lower energy has spin $+1 / 2$ and transforms as $\Gamma_{4}\left(\overline{\mathbf{C}}_{3}\right)$, while its counterpart with spin $-1 / 2$ transforms as $\Gamma_{5}\left(\overline{\mathrm{C}}_{3}\right)$. The $\Gamma_{56}\left(\overline{\mathrm{C}}_{3 v}\right)$ states also split into two states, both transforming as $\Gamma_{6}\left(\overline{\mathrm{C}}_{3}\right)$. The selection rules for dipole transitions then require spin flip in $\overline{\mathrm{C}}_{3}$ symmetry, as long as photon polarization $\mathbf{E} \perp \mathbf{c}$ is considered, with either LCP or RCP. Transitions between the $\Gamma_{6}\left(\overline{\mathrm{C}}_{3}\right)$ components into which the original $\Gamma_{56}\left(\overline{\mathrm{C}}_{3 v}\right)$ states split in magnetic field $\mathbf{B} \| \mathbf{c}$ are also allowed, but with $\mathbf{E} \| \mathbf{c}$ polarization, which is not observed in our experimental geometry for $\mathbf{B} \| \mathbf{c}$. In principle, transition between the $\Gamma_{6}\left(\overline{\mathbf{C}}_{3}\right)$ levels of the excited state and $\Gamma_{4}\left(\overline{\mathrm{C}}_{3}\right)$ or $\Gamma_{5}\left(\overline{\mathrm{C}}_{3}\right)$ sublevels of the ground state and vice versa are also allowed with either RCP or LCP polarization; however, such transitions are not observed in our experiment, probably because they stem from the $\mathrm{Nb}_{1}$ line polarized $\mathbf{E} \| \mathbf{c}$ without magnetic field. Its components in magnetic field $\mathbf{B} \| \mathbf{c}$ up to $5 \mathrm{~T}$ may remain too weak (or remain obscured within the strong $\Gamma_{5} \leftrightarrow \Gamma_{4}$ transitions) and are not discerned in the $\mathbf{E} \perp \mathbf{c}$ polarization inherent to Faraday geometry. We notice that in general, while our models based on consideration of $p$-like hole provide quite good description of the observed Zeeman splitting of the lines, they do not provide the expected understanding for the polarization of all the lines (observed only for the case $\mathbf{B} \| \mathbf{c}$ in this work), possibly due to neglect of mixing of any other but $p$-like states in our model Hamiltonians, as well as neglect of geometrical factors which might influence the measurement.

In what concerns the case $\mathbf{B} \perp \mathbf{c}$, due to the low symmetry $\left(\overline{\mathrm{C}}_{1}\right)$ all transitions are allowed from a group-theoretical point of view. However, the group theory does not allow us to deduce the oscillator strength of the possible transitions; neither can we rely on our simple models for this purpose. Therefore, comparison between the models and the experiment in this case will be carried out on a semiqualitative basis by considering the behavior of the photon energy vs magnetic field dependence of those transitions which we believe dominate the experimentally observed spectra.

\section{Comparison with experiment}

Let us start with quantitative comparison of the two models. In the case $\mathbf{B} \| \mathbf{c}$, the Hamiltonian within the $j$ model remains diagonal also for $\mathbf{B} \neq \mathbf{0}\left[B_{\|} \neq 0, B_{\perp}=0\right.$; cf. Eqs. (9) and (10)], hence it is easy to see that all states with $m_{j}=\frac{1}{2}$ split as $\pm \frac{\mu B_{\|}}{2}$, whereas the states with $m_{j}=\frac{3}{2}$ split as $\pm \frac{3 \mu B_{\|}}{2}$ (valid for both the ground and excited states). This picture is in agreement with our previous intuitive consideration of spin$1 / 2$ splitting and leads to splitting of the PL lines in the case B $\|$ c by amount $\approx 2 \mu B_{\|}$, which will be seen to agree with the experimental data. On the other hand, for the same situation
(B $\|$ c) the $l s$ model predicts for the excited state that the $\Gamma_{56}$ state splits as $\pm 2 \mu B_{\|}$, whereas the splitting of the two mixing $\Gamma_{4}$ states is strongly dependent on the relation between $\lambda_{\|}$and $\lambda_{\perp}$. If we were to deal with the situation $\left|\lambda_{\perp}\right| \ll\left|\lambda_{\|}\right|$ (or the extreme $\lambda_{\perp}=0$ ), we would obtain that the $\Gamma_{4}$ state intermediate in energy does not split at all in magnetic field B $\| \mathbf{c}$, whereas the other $\Gamma_{4}$ state (highest in energy) splits as $\pm \mu B_{\|}$, a situation which disagrees with the experimental data. On the contrary, admitting $\left|\lambda_{\perp}\right| \gg\left|\lambda_{\|}\right|$[as in Figs. 6(b) and 6(d)] we obtain that now both $\Gamma_{4}$ states split by an amount close to $\pm \frac{\mu B_{\|}}{2}$, similar to the situation in the $j$ model. Thus, strong mixing of the $\Gamma_{4}$ states (induced by large value of $\left|\lambda_{\perp}\right| \gg$ $\left.\left|\lambda_{\|}\right|\right)$leads to similar behavior of the $\Gamma_{4}$ states in the $j$ and ls models in magnetic field $\mathbf{B} \| \mathbf{c}$. This stipulates the choice of $\lambda_{\perp}=-1.270 \mathrm{meV}$ (close to the maximum value for $\left|\lambda_{\perp}\right|$ ) made earlier for the plot shown in Fig. 6.

Let us compare the prediction of the two models for the excited state ( $j$ model for the ground state) with the experimental data. Considering first the case $\mathbf{B} \| \mathbf{c}$ we notice from Figs. 6(a) and 6(b) that both models predict nearly linear dependence of the energy levels on the magnetic field, and in both models the $\Gamma_{4}$ levels split by an amount close to $\pm \frac{\mu B_{\|}}{2}$. However, whereas this splitting is exactly $\pm \frac{\mu B_{\|}}{2}$ in the $j$ model, there is small asymmetry in the splitting of the two $\Gamma_{4}$ components of the excited state within the $l s$ model: the lower-in-energy $\Gamma_{4}$ state splits slightly less than $\pm \frac{\mu B_{\|}}{2}$, while the uppermost one splits slightly more, as seen in Fig. 6(b). Since within the Faraday geometry for the case $\mathbf{B} \| \mathbf{c}$ we anticipate mainly transitions involving $\Gamma_{4}$ and $\Gamma_{5}$ states (within $\overline{\mathrm{C}}_{3}$ ), the above-mentioned asymmetry will result in slightly different splitting between the $\mathrm{Nb}_{0}$ and $\mathrm{Nb}_{2}$ lines. However, within the experimental error the splitting with magnetic field B || $\mathbf{c}$ for these two lines is the same. This is illustrated in Fig. 7(a), where the lines represent the calculated transition energies using the $j$ model for the transitions denoted by arrows in Fig. 6(a). Therefore, the $j$ model provides almost exact description of the experimental data for $\mathbf{B} \| \mathbf{c}$, although the $l s$ model (with $\lambda_{\perp}=-1.270 \mathrm{meV}$ ) is also in reasonable agreement with it. The $j$ model predicts splitting of both PL lines by $2 \mu B_{\|}$exactly, to be compared with $\sim 1.9 \mu B_{\|}$observed experimentally (cf. Fig. 4), but this slight discrepancy may be attributed to slight (uncontrolled) misorientation between the $c$ axis and the direction of the magnetic field. All allowed transitions are denoted with arrows in Fig. 6(a) and occur symmetrically shifted at energies $\pm \mu B_{\|}$with respect to the corresponding zero-field line positions.

As seen from Fig. 4, we observe contributions from both circular polarizations in all lines. Note however, that the low-energy component of the $\mathrm{Nb}_{0}$ and $\mathrm{Nb}_{3}$ lines is dominated by $\mathrm{RCP}$, but for $\mathrm{Nb}_{2}$ the low-energy component has predominantly the opposite LCP. The exact determination of the peak positions for the $\mathrm{Nb}_{5}$ line from the spectra is hindered due to its weakness and overlap with the stronger $\mathrm{Nb}_{3}$ line, but its behavior is clearly analogous to that of $\mathrm{Nb}_{2}$ : the low-energy component is dominated by LCP and the high-energy one by RCP. Thus, the polarization and behavior in magnetic field $\mathbf{B} \| \mathbf{c}$ is analogous, on one hand, for the pair of lines $\mathrm{Nb}_{0}$ and $\mathrm{Nb}_{3}$, and, on the other hand, for the pair $\mathrm{Nb}_{2}$ and $\mathrm{Nb}_{5}$. This analogy can be complemented with the same 

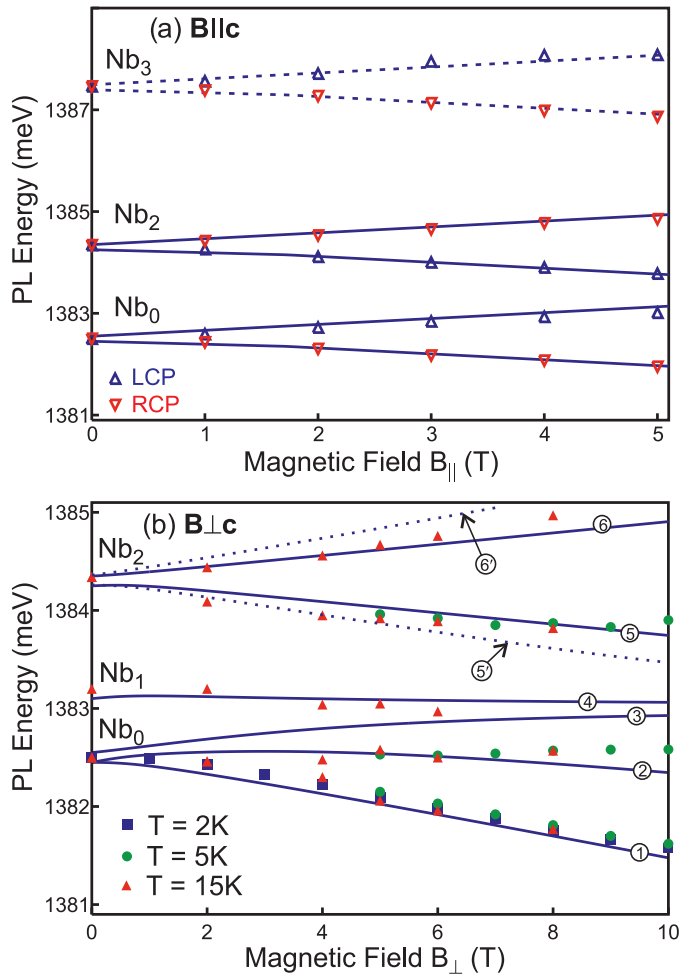

FIG. 7. (Color online) Comparison of all experimental data with the phenomenological models for the cases (a) $\mathbf{B} \| \mathbf{c}$ and (b) $\mathbf{B} \| \mathbf{c}$. (Most of the spectra are shown in Figs. 4 and 5.) The symbols in the panels represent the experimental line positions. The full lines are the theoretically calculated line positions using the $j$ model for the ground and excited states. The dashed lines in part (a) are obtained by shifting the full lines from $\mathrm{Nb}_{0}$ to $\mathrm{Nb}_{3}$, demonstrating the same splitting of these lines. The dotted lines in part (b) represent transitions denoted $5^{\prime}$ and $6^{\prime}$ in Fig. 6(d), which are calculated using the $l s$ model for the excited state and the $j$ model for the ground state, and are given for comparison. Note that three different symbols are used to denote the data from the spectra measured at the three different temperatures in the case $\mathbf{B} \perp \mathbf{c}$, and the enumeration of the theoretical lines follows the enumeration of the transitions in Fig. 6.

polarization $(\mathbf{E} \| \mathbf{c})$ of the middle lines in the triplets, $\mathrm{Nb}_{1}$ and $\mathrm{Nb}_{4}$, in absence of magnetic field. Thus, the high-energy triplet has similar optical properties to the low-energy one, which corroborates our concept that the high-energy triplet also stems from the valence-band threefold splitting inherited by the weakly bound hole in the $\mathrm{Nb}$-bound exciton, but when the center is in $\mathrm{C}_{1 h}$ symmetry, as already discussed in Sec. IV E.

The reason for the opposite polarization (LCP vs RCP) of, e.g., the low- or high-energy components of $\mathrm{Nb}_{0}$ and $\mathrm{Nb}_{2}$ are not fully understood at present. This model predicts that all low-energy branches will have LCP, while the high-energy ones will have RCP. Thus, the model is in agreement with the dominant polarization for $\mathrm{Nb}_{0}$ (and $\mathrm{Nb}_{3}$ ) only; the opposite behavior of $\mathrm{Nb}_{2}$ and $\mathrm{Nb}_{5}$ is not understood at present.

Let us consider now the case $\mathbf{B} \perp \mathbf{c}$. The splitting of the ground state $(j$ model) remains approximately linear. However, the magnetic field $\mathbf{B} \perp \mathbf{c}$ induces strong mixing between the substates of the excited state (with the exception of the highest-energy doublet in the $j$ model) leading to nonlinear dependence of the energies of the interacting states on the magnetic field. Also, in this case due to low symmetry basically all transitions are allowed, but our simple models do not allow calculation of the transition probabilities. Careful comparison of the predictions of the two models with the experimental data allows us to conclude that the $j$ model provides overall better description of the experiment. In Fig. 7(b) we have summarized the experimental line positions at different temperatures (symbols) and plotted the transition-energy dependencies calculated using the $j$ model for those transitions which are probably dominating the PL spectra. These transitions are also depicted by arrows in Fig. 6(c). The choice of the depicted transitions is consistent with the temperature dependence of the PL spectra shown in Fig. 5. Thus, for example, at the lowest temperature $(T=2 \mathrm{~K})$ only one PL line is observed, which stems from transition involving the lowest in energy branch of the excited state. Comparison of the slope of the experimental field dependence of the line position with the four possible slopes corresponding to transitions from this branch to the four branches of the ground state indicates that one of them has dominating transition probability [denoted 1 in Fig. 6(c)], because this is the only transition that reproduces very well the slope observed experimentally. We note that no good agreement with the experimental data for this transition can be achieved if the $l s$ model is used for the excited state, due to the different slope of the lowermost curve in the excited state [cf. Figs. 6(c) and 6(d)]. Similarly, at slightly higher temperature $(T=5 \mathrm{~K})$ transitions involving higher excited states can be observed experimentally, mainly involving the two branches of the lowest in energy $\Gamma_{4}$ state and the lower branch of the highest-in-energy $\Gamma_{4}$ state of the excited state [denoted by 2 and 3 in Fig. 6(c)]. As already noticed, the components of the $\mathrm{Nb}_{1}$ line polarized $\| \mathbf{c}$ remain very weak in this experimental geometry, which we attributed in Sec. V A to its different polarization $(\| \mathbf{c})$ and the fact that the luminescence is detected using a fiber. Thus, one component of the $\mathrm{Nb}_{1}$ line becomes visible [albeit weakly; denoted as 4 in Fig. 6(c)] only at $T=15 \mathrm{~K}$. At this temperature $(15 \mathrm{~K})$ transition also from the higher branch of the high-energy $\Gamma_{4}$ state can be discerned, too [denoted 6 in Fig. 6(c)]. The agreement between the experimental data and the calculated PL line positions within the $j$ model presented in Fig. 7 is quite reasonable. We notice, however, that the $j$ model does not describe the nonlinearity in the field dependence of the highest-energy PL lines, which can be seen in Fig. 7(b) for $\mathbf{B} \perp \mathbf{c}$. This is due to the fact that in the frame of the $j$ model the highest-in-energy $\Gamma_{4}$ state of the excited state does not mix with the rest of the states. With the choice of $\lambda_{\perp}=-1.270 \mathrm{meV}$ the $l s$ model reproduces better the nonlinearity of the higher-energy dependencies [the corresponding transitions are denoted $5^{\prime}$ and $6^{\prime}$ in Fig. 6(d)]; these transitions are displayed with dotted lines in Fig. 7(b) (enumerated $5^{\prime}$ and $6^{\prime}$ ) for comparison with the prediction of the $j$ model displayed with full lines. It is worth noting that the observed nonlinearity of the highest-energy lines can be described better within the $l s$ model if all transitions to the ground-state manifold are considered (not shown). However, we have refrained from fitting the parameters for this purpose in view of the simplicity of the model. To conclude, the $j$ and $l s$ models together provide quite reasonable description of the 
Zeeman data, in accord with the concept of the $\mathrm{Nb}$-bound exciton presented in Sec. III.

\section{CONCLUSIONS}

The presented model of a bound exciton with strongly bound electron and weakly bound (effective-mass-like) hole provides a quite detailed explanation of the experimental data presented. The experimentally observed energy of the $\mathrm{Nb}$-bound exciton in $4 \mathrm{H}-\mathrm{SiC}$ is in very good agreement with the theoretical results obtained from first principles, which demonstrate also the trend of quenching of the crystal-field splitting of the weakly bound hole, in agreement with the experimental observation. The gross features of the Zeeman spectra can be understood on the basis of a simplified model using phenomenological Hamiltonian in order to describe the splitting of the components of the ground and excited states in the PL transition. Finally, we note that our model implies that $\mathrm{Nb}$ in the ASV $h-h$ configuration acts as a deep acceptor.

\section{ACKNOWLEDGMENTS}

The Knut and Alice Wallenberg Foundation is acknowledged for support. NSC supercomputer time is acknowledged. In addition, A.G. acknowledges the Lendület program of the Hungarian Academy of Sciences, and the support of the Hungarian OTKA Project No. K101819. I.A.A. acknowledges the support from the Ministry of Education and Science of the Russian Federation (Grant No. 14.Y26.31.0005) and the Tomsk State University Academic D. I. Mendeleev Fund Program (Project No. 8.1.18.2015).
[1] J. Schneider, H. D. Müller, K. Maier, W. Wilkening, F. Fuchs, A. Dörnen, S. Leibenzeder, and R. Stein, Appl. Phys. Lett. 56, 1184 (1990).

[2] B. Kaufmann, A. Dörnen, and F. S. Ham, Phys. Rev. B 55, 13009 (1997).

[3] J. Reinke, H. Weihrich, S. Greulich-Weber, and J.-M. Spaeth, Semicond. Sci. Technol. 8, 1862 (1993).

[4] Y. Koshka, M. Mazzola, S. Yingquan, and C.U. Pittman, Jr., J. Electron. Mater. 30, 220 (2001).

[5] J. Baur, M. Kunzer, and J. Schneider, Phys. Status Solidi A 162, 153 (1997).

[6] N. T. Son, A. Ellison, B. Magnusson, M. F. MacMillan, W. M. Chen, B. Monemar, and E. Janzěn, J. Appl. Phys. 86, 4348 (1999).

[7] L. Patrick and W. J. Choyke, Phys. Rev. B 10, 5091 (1974).

[8] A. W. C. van Kemenade and S. H. Hagen, Solid State Commun. 14, 1331 (1974).

[9] A. Henry, J. P. Bergman, and E. Janzén, Mater. Sci. Forum 600, 461 (2009).

[10] A. Gällström, B. Magnusson, F. C. Beyer, A. Gali, N. T. Son, S. Leone, I. G. Ivanov, C. G. Hemmingsson, A. Henry, and E. Janzén, Physica B 407, 1462 (2012).

[11] K. F. Dombrowski, M. Kunzer, U. Kaufmann, J. Schneider, P. G. Baranov, and E. N. Mokhov, Phys. Rev. B 54, 7323 (1996).

[12] M. Kunzer, K. F. Dombrowski, F. Fuchs, U. Kaufmann, J. Schneider, P. G. Baranov, and E. N. Mokhov, Inst. Phys. Conf. Ser. 142, 385 (1996).

[13] A. Gällström, B. Magnusson, and E. Janzén, Mater. Sci. Forum 615, 405 (2009).

[14] B. Magnusson and E. Janzén, Mater. Sci. Forum 483, 341 (2005).

[15] Mt. Wagner, B. Magnusson, W. M. Chen, and E. Janzén, Phys. Rev. B 66, 115204 (2002).
[16] V. Ivády, A. Gällström, N. T. Son, E. Janzén, and A. Gali, Phys. Rev. Lett. 107, 195501 (2011).

[17] N. T. Son, X. T. Trinh, A. Gällström, S. Leone, O. Kordina, E. Janzén, K. Szász, V. Ivády, and A. Gali, J. Appl. Phys. 112, 083711 (2012).

[18] I. G. Ivanov, A. Gällström, S. Leone, O. Kordina, N. T. Son, A. Henry, V. Ivády, A. Gali, and E. Janzén, Mater. Sci. Forum 740, 405 (2013).

[19] O. Kordina, C. Hallin, A. Ellison, A. S. Bakin, I. G. Ivanov, A. Henry, R. Yakimova, M. Touminen, A. Vehanen, and E. Janzén, Appl. Phys. Lett. 69, 1456 (1996).

[20] L. Patrick, D. R. Hamilton, and W. J. Choyke, Phys. Rev. 132, 2023 (1963).

[21] R. P. Devaty, S. Bai, W. J. Choyke, D. Hobgood, and D. J. Larkin, Mater. Sci. Forum 353, 357 (2001).

[22] F. Zhang, Y. Gao, H. Cui, X. Ruan, and W. Zhang, Adv. Mater. Res. 971, 77 (2014).

[23] N. T. Son, V. Ivády, A. Gali, A. Gällström, S. Leone, O. Kordina, and E. Janzén, Mater. Sci. Forum 717, 217 (2012).

[24] C. Persson and U. Lindefelt, J. Appl. Phys. 82, 5496 (1997).

[25] G. F. Koster, J. O. Dimmock, R. G. Wheeler, and H. Statz, Properties of the Thirty-Two Point Groups (MIT Press, Cambridge, MA, 1963).

[26] A. Gali, E. Janzén, P. Deák, G. Kresse, and E. Kaxiras, Phys. Rev. Lett. 103, 186404 (2009).

[27] V. Ivády, I. A. Abrikosov, E. Janzén, and A. Gali, Phys. Rev. B 87, 205201 (2013).

[28] P. E. Blöchl, Phys. Rev. B 50, 17953 (1994).

[29] G. Kresse and J. Furthmuller, Phys. Rev. B 54, 11169 (1996).

[30] S. Lany and A. Zunger, Phys. Rev. B 80, 085202 (2009).

[31] I. Dabo, A. Ferretti, N. Poilvert, Y. Li, N. Marzari, and M. Cococcioni, Phys. Rev. B 82, 115121 (2010).

[32] C. Freysoldt, J. Neugebauer, and C. G. Van de Walle, Phys. Rev. Lett. 102, 016402 (2009). 